\title{
Characterization, expression patterns and functional analysis of the MAPK and MAPKK genes in watermelon (Citrullus lanatus)
}

Qiuming Song ${ }^{1}$, Dayong Li', Yi Dai', Shixia Liu', Lei Huang ${ }^{1}$, Yongbo Hong ${ }^{1}$, Huijuan Zhang ${ }^{1,2^{*}}$ and Fengming Song ${ }^{1}$

\begin{abstract}
Background: Mitogen-activated protein kinase (MAPK) cascades, which consist of three functionally associated protein kinases, namely MEKKs, MKKs and MPKs, are universal signaling modules in all eukaryotes and have been shown to play critical roles in many physiological and biochemical processes in plants. However, little or nothing is known about the MPK and MKK families in watermelon.

Results: In the present study, we performed a systematic characterization of the CIMPK and CIMKK families including the identification and nomenclature, chromosomal localization, phylogenetic relationships, CIMPK-CIMKK interactions, expression patterns in different tissues and in response to abiotic and biotic stress and transient expression-based functional analysis for their roles in disease resistance. Genome-wide survey identified fifteen CIMPK and six CIMKK genes in watermelon genome and phylogenetic analysis revealed that both of the CIMPK and CIMKK families can be classified into four distinct groups. Yeast two-hybrid assays demonstrated significant interactions between members of the CIMPK and CIMKK families, defining putative CIMKK2-1/CIMKK6-CIMPK4-1/ CIMPK4-2/CIMPK13 and CIMKK5-CIMPK6 cascades. Most of the members in the CIMPK and CIMKK families showed differential expression patterns in different tissues and in response to abiotic (e.g. drought, salt, cold and heat treatments) and biotic (e.g. infection of Fusarium oxysporum f. sp. niveum) stresses. Transient expression of CIMPK1, CIMPK4-2 and CIMPK7 in Nicotiana benthamiana resulted in enhanced resistance to Botrytis cinerea and upregulated expression of defense genes while transient expression of CIMPK6 and CIMKK2-2 led to increased susceptibility to $B$. cinerea. Furthermore, transient expression of CIMPK7 also led to hypersensitive response (HR)-like cell death and significant accumulation of $\mathrm{H}_{2} \mathrm{O}_{2}$ in N. benthamiana.
\end{abstract}

Conclusion: We identified fifteen CIMPK and six CIMKK genes from watermelon and analyzed their phylogenetic relationships, expression patterns and protein-protein interactions and functions in disease resistance. Our results demonstrate that CIMPK1, CIMPK4-2 and CIMPK7 positively but CIMPK6 and CIMKK2-2 negatively regulate the resistance to $B$. cinerea when transiently expressed in N. benthamiana and that CIMPK7 functions as a regulator of HR-like cell death through modulating the generation of $\mathrm{H}_{2} \mathrm{O}_{2}$.

Keywords: Watermelon (Citrullus lanatus), Mitogen-activated protein kinase cascade, CIMPK, CIMKK, Protein-protein interaction, Expression patterns, Transient expression, Disease resistance

\footnotetext{
* Correspondence: zhanghj82@zju.edu.cn

${ }^{1}$ State Key Laboratory for Rice Biology, Institute of Biotechnology, Zhejiang University, Hangzhou 310058, P. R. China

${ }^{2}$ College of Life Science, Taizhou University, Taizhou, Zhejiang 318001, P. R.

China
} 


\section{Background}

Mitogen-activated protein kinase (MAPK) cascades, which are widely distributed in eukaryotes, are highly conserved signaling modules downstream of receptors/ sensors that transduce extracellular stimuli into intracellular responses $[1,2]$. The MAPK cascades are composed of three sequentially acting protein kinases, namely MAPKK kinases (MEKKs), MAPK kinases (MKKs) and MAPKs (MPKs), and activated through the way of phosphorylation $[1,3]$. In general, upon perception of the extracellular environmental and intracellular growth/developmental signals, the top kinases of the cascades, MEKKs, activate via phosphorylation their downstream MKKs, which in turn further phosphorylate MPKs [4]. In specific, the MKKs in the MAPK cascades act as dual-specificity kinases to activate MPKs through double phosphorylation of the $\mathrm{T}-\mathrm{x}-\mathrm{Y}$ motif in the activation loop. During this phosphorylation relay, the input signal can be amplified through the MAPK cascade and eventually the activated MAPKs modify via phosphorylation a set of specific downstream target proteins such as transcription factors and other signaling components leading to the activation of the expression of downstream genes $[1,4,5]$.

During the last two decades, extensive genetic and biochemical studies have been performed to explore the functions of MAPK cascades in model plant species as well as in some economically important crops such as rice. These studies have demonstrated that the MAPK cascades and their individual components play critical roles in regulating growth/development and stress responses in plants. Furthermore, several functional intact MAPK cascades that are involved in growth/development and stress responses have been characterized biochemically $[1,2,4]$. For example, tobacco NPK1NQK1-NRK1 and Arabidopsis YODA-MKK4/MKK5MPK3/MPK6 play essential roles in cell division, whereas Arabidopsis MEKK1-MKK4/MKK5 -MPK3/ MPK6 and MEKK1-MKK1/2-MPK4 act as positive or negative regulators of signaling pathways modulating the immune responses $[1,2,6,7]$.

The components of the MAPK cascades are generally composed of different gene families, namely MPK, MKK and MEKK families, which have been characterized at the genome-wide level in many plant species including Arabidopsis [8, 9], rice [9, 10], poplar [9], soybean [11], maize [12, 13], tomato [14-16], canola [17], banana [18], apple [19], Gossypium raimondii [20], mulberry [21] and Brachypodium distachyon [22]. The numbers of MPK and MKK families vary greatly across species. For example, there are $20 \mathrm{MPKs}$ in Arabidopsis [8, 9], 17 in rice [9, 10], 19 in maize [13], 21 in poplar [9], 16 in tomato [14], 12 in canola [17], 10 in mulberry [21], 12 in grapevine [23], 17 in tobacco [24], 38 in soybean [11], 28 in G. raimondii [20] and 16 in B. distachyon [22]. Similarly, $10 \mathrm{MKKs}$ in Arabidopsis [8, 9], 8 in rice [9], 9 in maize [12], 5 in tomato [15, 16] and in canola [17], 11 in soybean [11], 11 in poplar [9], and 12 in B. distachyon [22] were identified. Structurally, the MPKs contain eleven domains (I-XI) and the well conserved threonine and tyrosine residues existing between domains VII and VIII form the activation loop, which is thought to be phosphorylated for the activation of the MPKs [25]. It is well known that plant MPKs have two different activation loop motifs, either TEY or TDY; however, other novel activation loop variants were recently characterized in plants MPKs [26]. Generally, the MPKs can be divided into four groups based on phylogeny and the conserved TEY/TDY motifs and each group has been assigned different functions [8, 27]. Similarly, the MKKs can also be classified into four groups according to the S/T-x5-S/T domain and "D site" [8].

Watermelon (Citrullus lanatus) is one of important horticultural crops, providing favorite fresh fruits worldwide. However, little or nothing is known about the MPK and MKK families in watermelon so far. The recently completion of genome sequencing of watermelon [28] provides a powerful platform that makes it possible to characterize gene families at the genome-wide level. In the present study, we performed a genome-wide identification of the watermelon MPK and MKK families and carried out an extensive characterization of the CIMPK and ClMKK families in terms of the nomenclature, chromosomal distribution, the conserved motifs and phylogenetical relationships. We explored some selected members of the CIMPK and ClMKK families for their putative protein-protein interaction relationships, expression patterns among different tissues and in response to abiotic and biotic stresses and possible functions in disease resistance through transient expression-based functional analysis in Nicotiana benthamiana. Our characterization of the watermelon ClMPK and ClMKK families provides a useful platform for further functional studies of CIMPKs and ClMKKs in watermelon.

\section{Results \\ Characterization of the CIMPK and CIMKK families in watermelon}

To identify putative MPK and MKK genes in watermelon, we performed BLAST searches against the watermelon genome database using the well-characterized Arabidopsis AtMPKs and AtMKKs as queries and identified 15 and 6 non-redundant sequences that are putative MPK and MKK genes, respectively. The predicted amino acid sequences of the putative ClMPKs and ClMKKs were further examined by ExPASy Proteomics Server for the presence of the characteristic conserved 
domains. Overall, our systematic analyses revealed that the CIMPK and CIMKK families comprise of 15 and 6 members in the watermelon genome, respectively. For convenience, we assigned unique identities to each of the identified CIMPK and ClMKK genes with a twoletter code corresponding to $C$. lanatus $(\mathrm{Cl})$, followed by the family name (MPK or MKK) and a number (Table 1) according to the Arabidopsis MPK and MKK nomenclature system [8]. Notably, the predicted loci Cla022002 (402 bp) and Cla022003 (867 bp), which are exactly the same to the loci CL08G09900 and CL08G09910 in PLAZA dicots 3.0 database (http://bioinformatics.psb.ugent.be/plaza/), were indeed the same gene encoding for CIMPK6 and encode polypeptides corresponding for 1121 aa and 122-395 aa of AtMPK6. The coding sequence of ClMPK6 was further confirmed by our cloning of the full-length cDNA using primers designed according to the predicted cDNA sequences of Cla022002 and Cla022003.

To assess whether the characterized ClMPK and $C l M K K$ genes had expression support, we searched using the predicted cDNA sequences as queries against watermelon EST database (http://www.icugi.org/cgi-bin/ ICuGI/tool/blast.cgi). The search results indicated that 14. ClMPK and 2 ClMKK genes had available EST supports (Table 1), representing 93.3 and $33.3 \%$ of the
ClMPK and ClMKK genes, respectively. We attempted to clone the full-length cDNAs of all ClMPKs and $C l M K K s$ for the confirmation of the predicted sequences and for the functional and protein-protein interaction studies. However, we failed to amplify the full-length cDNAs for ClMPK9-1, ClMPK9-3, ClMPK9-4, ClMPK20-1 and ClMPK20-2, which have EST supports, and for ClMKK3 and ClMKK9, which do not have EST supports (Table 1). Ultimately, we amplified and cloned $10 \mathrm{ClMPK}$ and $4 \mathrm{ClMKK}$ genes, including ClMPK13, ClMKK2-1 and ClMKK6 that do not have EST supports (Table 1), for further studies in protein-protein interactions and functional analyses.

The sizes of the open reading frames (ORF) for the ClMPK genes range from $1107 \mathrm{bp}$ (ClMPK7) to $1926 \mathrm{bp}$ (ClMPK9-1) and accordingly the sizes of the encoded proteins range from 368 to 641 amino acids. The molecular weights of the CIMPK proteins are between $42.57 \mathrm{kD}$ and $72.87 \mathrm{kD}$ and the pIs range from 4.97 to 9.37 (Table 1). The predicted ClMKK9 is likely an incomplete MKK and lacks approximately 100 amino acids at the N-terminal when compared with its closest Arabidopsis homologue AtMKK9. The ORF sizes for the other five ClMKK genes range from 1023 bp (ClMKK2-2) to 1557 bp (ClMKK3) and accordingly the sizes of the encoded proteins range from 340 to 518 amino acids.

Table 1 Information on CIMPKs and CIMKKs in watermelon

\begin{tabular}{|c|c|c|c|c|c|c|c|c|c|c|}
\hline Family & Genes & Loci & ORF (bp) & Size (aa) & $\mathrm{MW}(\mathrm{kD})$ & $p l$ & T-loop & Group & EST no. & Full cDNA \\
\hline \multirow[t]{15}{*}{ MPK } & CIMPK1 & $\mathrm{Cla022470}$ & 1161 & 386 & 44.67 & 6.35 & TEY & $C$ & 1 & Yes \\
\hline & CIMPK3 & Cla008291 & 1899 & 632 & 71.58 & 5.41 & TEY & A & 3 & Yes \\
\hline & CIMPK4-1 & Cla011419 & 1152 & 383 & 44.01 & 6.47 & TEY & B & 3 & Yes \\
\hline & CIMPK4-2 & Cla006629 & 1140 & 379 & 43.74 & 6.13 & TEY & B & 1 & Yes \\
\hline & CIMPK6 & Cla022002+ Cla022003 & 1266 & 421 & 47.99 & 5.63 & TEY & A & 3 & Yes \\
\hline & CIMPK7 & Cla014573 & 1107 & 368 & 42.57 & 6.67 & TEY & C & 2 & Yes \\
\hline & CIMPK9-1 & Cla018932 & 1926 & 641 & 72.87 & 6.81 & TDY & $\mathrm{D}$ & 4 & - \\
\hline & CIMPK9-2 & Cla004511 & 1422 & 473 & 54.42 & 6.80 & TDY & $D$ & 2 & Yes \\
\hline & CIMPK9-3 & Cla003498 & 1422 & 473 & 54.50 & 7.27 & TDY & D & 1 & - \\
\hline & CIMPK9-4 & Cla018463 & 1554 & 517 & 59.19 & 8.44 & TDY & $D$ & 1 & - \\
\hline & CIMPK13 & Cla008298 & 1113 & 370 & 42.61 & 4.97 & TEY & B & - & Yes \\
\hline & CIMPK16 & Cla009366 & 1686 & 561 & 63.85 & 8.66 & TDY & $\mathrm{D}$ & 1 & Yes \\
\hline & CIMPK19 & Cla005389 & 1413 & 470 & 54.31 & 9.37 & TDY & $\mathrm{D}$ & 1 & Yes \\
\hline & CIMPK20-1 & Cla005523 & 1893 & 630 & 70.70 & 9.01 & TDY & $D$ & 4 & - \\
\hline & CIMPK20-2 & Cla013487 & 1848 & 615 & 69.76 & 9.21 & TDY & $\mathrm{D}$ & 2 & - \\
\hline \multirow[t]{6}{*}{ MKK } & CIMKK2-1 & Cla016842 & 1069 & 355 & 39.54 & 5.40 & - & A & - & Yes \\
\hline & CIMKK2-2 & Cla011187 & 1023 & 340 & 38.13 & 5.26 & - & A & 5 & Yes \\
\hline & CIMKK3 & Cla017119 & 1557 & 518 & 57.77 & 5.53 & - & B & - & - \\
\hline & CIMKK5 & Cla012564 & 1110 & 369 & 41.45 & 8.91 & - & C & 2 & Yes \\
\hline & CIMKK6 & Cla016802 & 1065 & 354 & 39.73 & 6.27 & - & A & - & Yes \\
\hline & CIMKK9 & Cla018437 & 636 & 211 & 23.60 & 6.23 & - & D & - & - \\
\hline
\end{tabular}




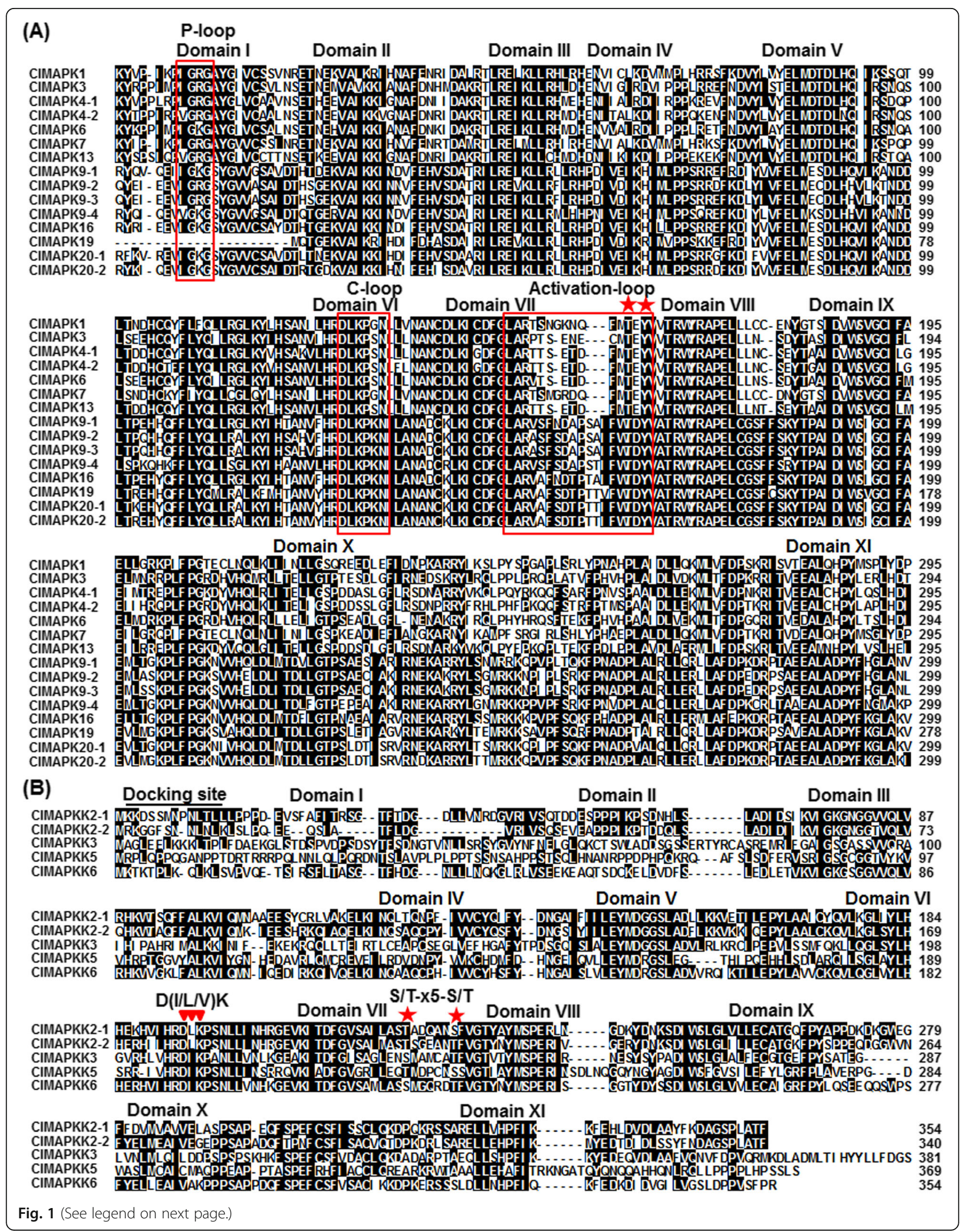


(See figure on previous page.)

Fig. 1 Sequence alignments and structural features of CIMPKs and CIMKKs. Multiple sequence alignment was performed using the ClustalX method and identical amino acids are shaded in black. The subdomains (I-XI) are indicated on the top of the aligned row. a Partial amino acid alignment of the 15 CIMPK proteins. The P-Loop, C-loop and activation-loop motifs are indicated with red boxes and the TxY motif is indicated by red stars. $\mathbf{b}$ Partial amino acid alignment of the 5 CIMKK proteins. The conserved S/T-x5-S/T motif and active site D(I/L/N)K motif are indicated by red stars and inverted red triangles, respectively. The docking site is indicated on the aligned row

The molecular weights of these CIMKK proteins are between $38.13 \mathrm{kD}$ and $57.77 \mathrm{kD}$ and the pIs range from 5.26 to 8.91 (Table 1 ).

\section{Structural features and phylogenetic analysis of the CIMPKs and CIMKKs}

Sequence alignment indicated that the ClMPK proteins contain highly conserved regions, spanning approximately 300 amino acids near the $\mathrm{N}$-terminal portion, which are composed of eleven characteristic domains (I-XI) (Fig. 1a). Phylogenetic tree analysis with Arabidopsis AtMPKs revealed that the ClMPKs can be divided into four groups, namely A, B, C and D (Fig. 2a). Among 15 ClMPKs, ClMPK3 and ClMPK6 belong to Group A, ClMPK4-1, ClMPK4-2 and ClMPK13 are Group B members, only CIMPK1 falls into Group $\mathrm{C}$, the other 8 members (ClMPK9-1, ClMPK9-2, CIMPK9-3, CIMPK94, CIMPK16, CIMPK19, CIMPK20-1 and CIMPK20-2) belong to Group D (Fig. 2a and Table 1). Several highly conserved characteristic motifs, e.g. activation-loop, Ploop and C-loop, were also identified in the CIMPK proteins (Fig. 1a). The activation-loop motifs are present between the domains VII and VIII and the TxY motif, which is phosphorylated for the activity, is present in all ClMPKs (Fig. 1a). Members in Groups A, B and C possess the TEY motif, whereas CIMPKs in Group D have the TDY motif (Fig. 1a and Table 1). However, no other TxY variant was found in all ClMPKs [14, 26]. In addition, a conserved $\mathrm{CD}$ domain with sequence of (LH)DxxDE(P)xC, which is thought to function as binding sites for upstream MKKs in the MAPK cascades [29], is present in Groups A and B ClMPKs but is absent in Group $\mathrm{C}$ and $\mathrm{D}$ ClMPKs. The TDY-containing CIMPKs have extended $\mathrm{C}$-terminal regions, which are generally present in the TDY class of MPKs from other plants $[8,14,18,22]$. In watermelon, there are 7 CIMPKs with TEY motif and 8 ClMPKs containing TDY motif (Table 1). This is similar to rice and $B$. distachyon, which contain more TDY-containing MPKs than the TEY-containing MPKs [9, 10, 22] but different from those in Arabidopsis, tomato, soybean and G. raimondii, which contain more TEY-containing MPKs than the TDY-containing MPKs [9, 11, 14, 20].

Sequence alignment revealed the ClMKKs except ClMKK9, which is an incomplete MKK, also contain 11 domains of protein kinases with serine/threonine specificity [9]. Conserved motifs were identified in ClMKKs.
The characteristic S/T-x5-S/T motif between domains VII and VIII, which includes the serine/threonine residues whose phosphorylation is necessary for MKK activation, and active site $\mathrm{D}(\mathrm{I} / \mathrm{L} / \mathrm{V}) \mathrm{K}$ motif were conserved in ClMKKs (Fig. 1b). In addition, putative docking regions with characteristic sequence of $K / R-K / R-K / R-x$ (1-6)-L-X-L/V/I were present in CIMKK2-1, ClMKK2-2, ClMKK3 and ClMKK6 (Fig. 1b). Phylogenetic tree analysis with Arabidopsis AtMKKs revealed that the ClMKKs can be divided into four groups, namely A, B, $\mathrm{C}$ and $\mathrm{D}$ (Fig. 2b). Among 6 ClMPKs, ClMKK2-1, ClMKK2-2 and CIMKK6 belong to Group A, whereas ClMKK3, ClMKK5 and ClMKK9 belong to Group B, C and $\mathrm{D}$, respectively (Fig. $2 \mathrm{~b}$ and Table 1 ). Similar to that in maize [12], the ortholog of AtMKK7/AtMKK8/ AtMKK9 was not found in watermelon (Fig. 2b). Furthermore, the ClMKK family is relatively smaller than other plant species such as Arabidopsis (10 AtMKKs) [8], rice (8 OsMKKs) [9], maize (9 ZmMKKs) [12], soybean (11 GmMKKs) [11]; popular (13 PtMKKS) [9] and B. distachyon (12 BdMKKs) [22]. The relatively small CIMKK family in watermelon may be a consequence from species-specific diversification during evolution and implies that the CIMKK proteins may have evolved to possess pleiotropic effects in diverse biological processes.

\section{Genomic distribution and evolution of the CIMPK and CIMKK families}

The $15 C l M P K$ and $6 C l M K K$ genes were anchored on ten of the 11 watermelon chromosomes (Fig. 3). The chromosomal distribution pattern indicated that some chromosomes and chromosomal regions have a relatively high density of ClMPK or ClMKK genes, e.g. neither ClMPK nor ClMKK gene was located on chromosome 5. In the ClMPK family, one ClMPK gene is located on each of chromosomes 1, 2, 4 and 9; two ClMPK genes were found to be located on chromosome 8 and three $C l M P K$ genes are distributed on each of the chromosomes 3, 6 and 7 (Fig. 3). In the ClMKK family,two ClMKK genes are located on chromosome 11 while only one $C l M K K$ gene is located on each of the chromosomes 3, 4, 7 and 10 (Fig. 3). No gene cluster, as defined by the criteria that four or more genes are present within a region of $200 \mathrm{~Kb}$ or less on a chromosome [29], was found for the ClMPK and ClMKK families. However, five paralog pairs such as ClMPK4-1/ 


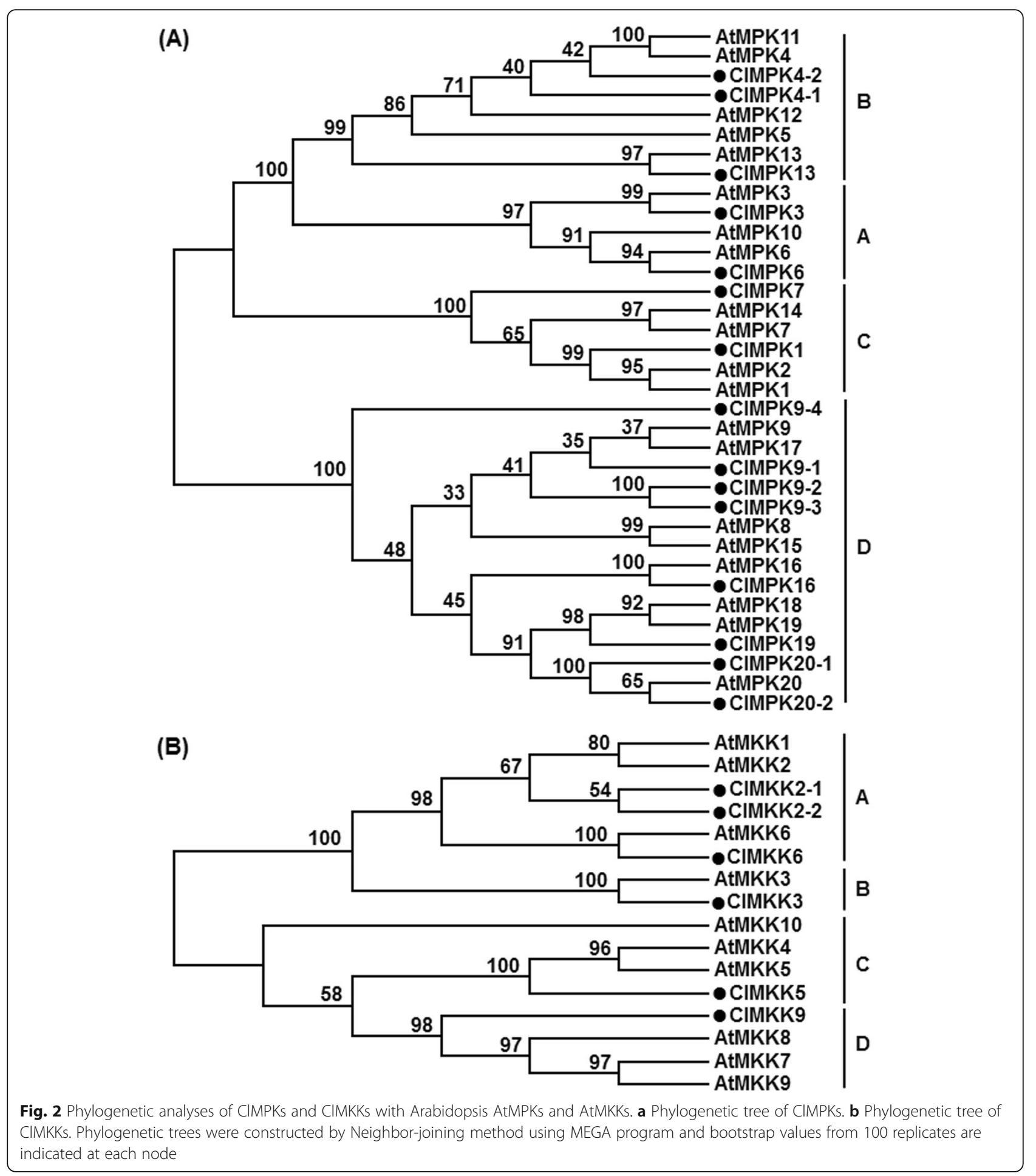

ClMPK4-2, ClMPK9-1/ClMPK9-4, ClMPK20-1/ClMP K20-2, ClMKK2-1/ClMKK2-2 and ClMKK2-2/ClMKK5, sharing high similarity in sequences, were distributed on different chromosomes (Fig. 3), indicating that they are not tandem duplicated gene pairs. Although ClMPK3 and ClMPK13 are tightly located on chromosome 3 , they only share $65 \%$ of identity at amino acid sequence level and are also not tandem duplicated genes. It is thus likely that tandem duplication plays a limited role in the evolution of the ClMPK and ClMKK genes. This is 


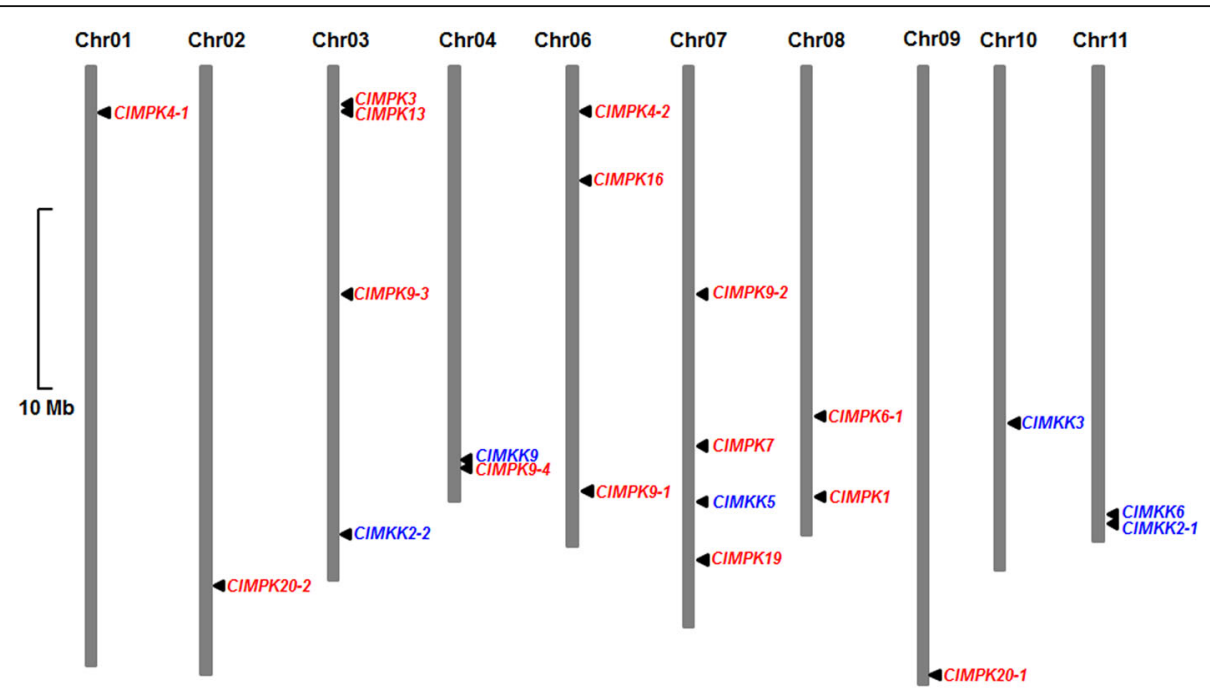

Fig. 3 Chromosomal distribution of the CIMPK and CIMKK genes. The CIMPK and CIMKK genes are indicated in red and blue colors, respectively. Scale bar represents $10 \mathrm{Mb}$

similar to the observations for the tomato SIMAPK and SlMKK families [14, 15].

\section{Interactions between CIMPKs and CIMKKs}

To examine the interactions and specificity between ClMPKs and ClMKKs, a series of yeast two-hybrid assays were performed to establish putative interaction relationships between ClMKKs and ClMPKs. For this purpose, four ClMKK genes (ClMKK2-1, ClMKK2-2, ClMKK5 and ClMKK6) and eight ClMPK genes (ClMPK1, ClMPK4-1, ClMPK4-2, ClMPK6, ClMPK7, ClMPK9-2, ClMPK13 and ClMPK16) were cloned into the respective DNA-binding domain and GAL4 activation domain plasmids, respectively. After co-transformation into the yeast strain YH2Gold, interactions were monitored by growth on selective medium and the production of blue pigment after addition of $\mathrm{X}$ - $\alpha$-gal. In our experiments, a positive control (pGADT7-T + pGBKT7-53) and a negative control (pGADT7-T + pGBKT7-Lam) were always included to rule out possible false interaction (Fig. 4a). As shown in Fig. 4b, interactions between tested ClMPKs and ClMKKs were detected. ClMKK2-1 exhibited strong interactions with CllMAPK4-2, ClMPK13 and ClMPK4-1; whereas ClMKK2-2 had a significant interaction with ClMPK1 (Fig. 4b). Similarly, significant interactions between ClMKK6 and CIMPK4-1, ClMPK4-2 or ClMPK13 and between ClMKK5 and ClMPK6-1 or ClMPK7 were observed (Fig. 4b). Among the ClMPKs tested, ClMPK9-2 and ClMPK16 were not found to interact with any of the four ClMKKs, probably having interactions with other ClMKKs.

\section{Expression patterns of CIMPK and CIMKK genes Tissue-specific expression patterns}

It is well known that MAPK cascades play critical roles in plants growth and development [2]. To gain insights into the involvement of the ClMPK and $C l M K K$ genes in growth and development, we analyzed by quantitative reverse transcription PCR (qRTPCR) their tissue-specific expression patterns in three different tissues such as roots, stems and leaves from 3-week-old watermelon plants. As shown in Fig. 5, the $15 C l M P K$ and $6 C l M K K$ genes were constitutively expressed in all tested tissues but exhibited different expression patterns. In the ClMPK family, ClMPK9-1, ClMPK1 and ClMPK7 in roots, ClMPK201, ClMPK3, ClMPK13, ClMPK4-2 and ClMPK6 in stems, and ClMPK9-3, ClMPK19, ClMPK16, ClMPK41, ClMPK9-4, ClMPK9-2 and ClMPK2O-2 in leaves showed the highest expression levels, whereas in the ClMKK family, the highest expression levels of ClMKK6 and ClMKK2-1 in roots, ClMKK2-2, ClMKK3 and ClMKK5 in stems and ClMKK9 in leaves were observed (Fig. 5). Comparison of the expression patterns identified some tissue-specifically expressed $C l M P K$ and $C l M K K$ genes, e.g., ClMPK3 having high expression level in stems but very low levels in roots and leaves, ClMPK7 with high expression level in roots but very low levels in stems and leaves, ClMPK19 showing high expression level in leaves but very low levels in roots and stems (Fig. 5a) and ClMKK5 having high expression level in stems but very low levels in roots and leaves (Fig. 5b), indicating that ClMPK3/ClMKK5, ClMPK7 and ClMPK19 


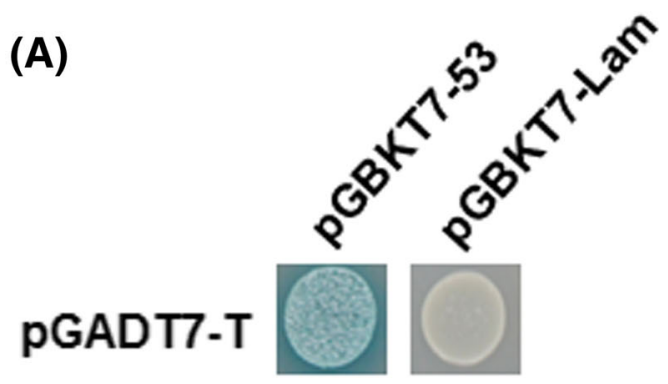

(B)

GADT7
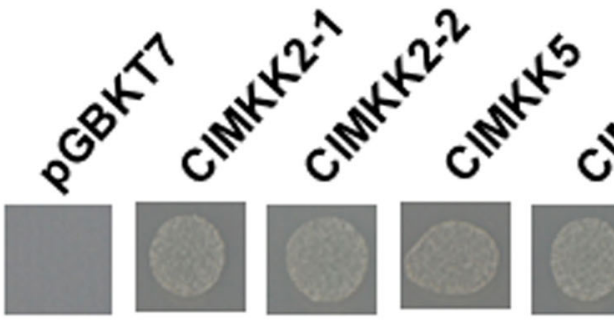

CIMPK1
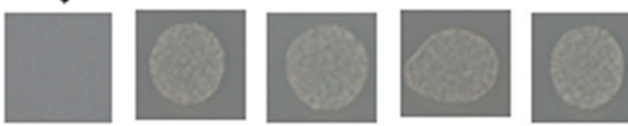

CIMPK4-1
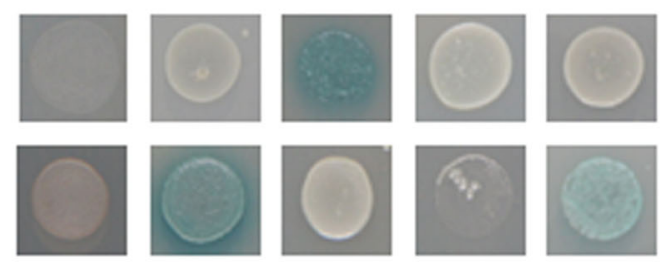

CIMPK4-2
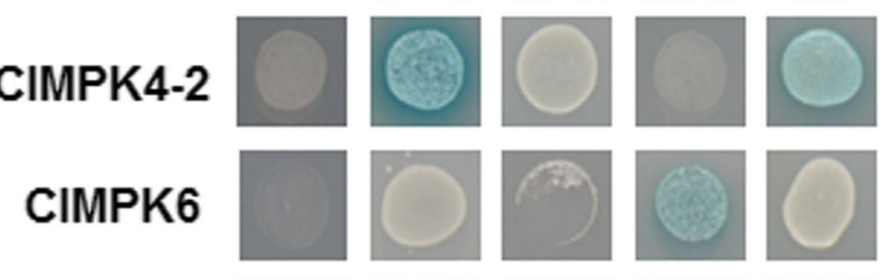

\section{CIMPK7}
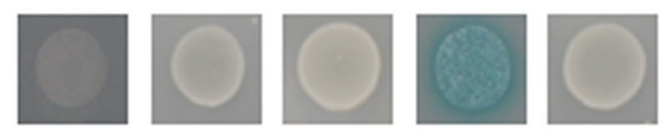

CIMPK9-2
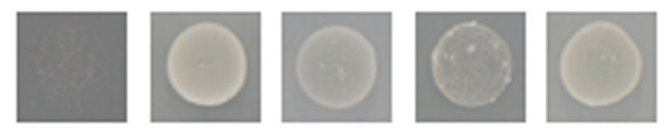

CIMPK13
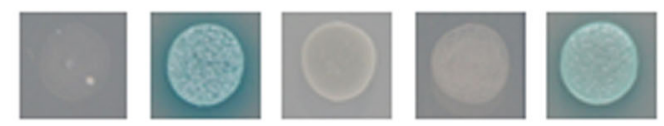

\section{CIMPK16}
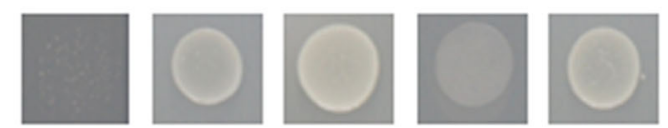

Fig. 4 Interactions between selected CIMPKs and CIMKKs. a Positive (pGADT7-T + pGBKT7-53) and negative (pGADT7-T + pGBKT7-Lam) controls. b Interactions between selected CIMPKs and CIMKKs. Yeasts harboring the indicated plasmid combinations were grown on selective medium SD/ $\mathrm{Trp}^{-} \mathrm{His}^{-}$and $\beta$-galactosidase activity showing positive interactions was examined by addition of X-a-gal. Repeated experiments showed similar results

may play specific roles in stems, roots and leaves, respectively. Furthermore, the paralog pairs ClMPK4-1/ ClMPK4-2, ClMPK9-1/ClMPK9-4, ClMPK20-1/ClMP
K20-2 and ClMKK2-1/ClMKK2-2, sharing high similarity in sequences, exhibited distinct expression patterns in roots, stems and leaves (Fig. 5), indicating 


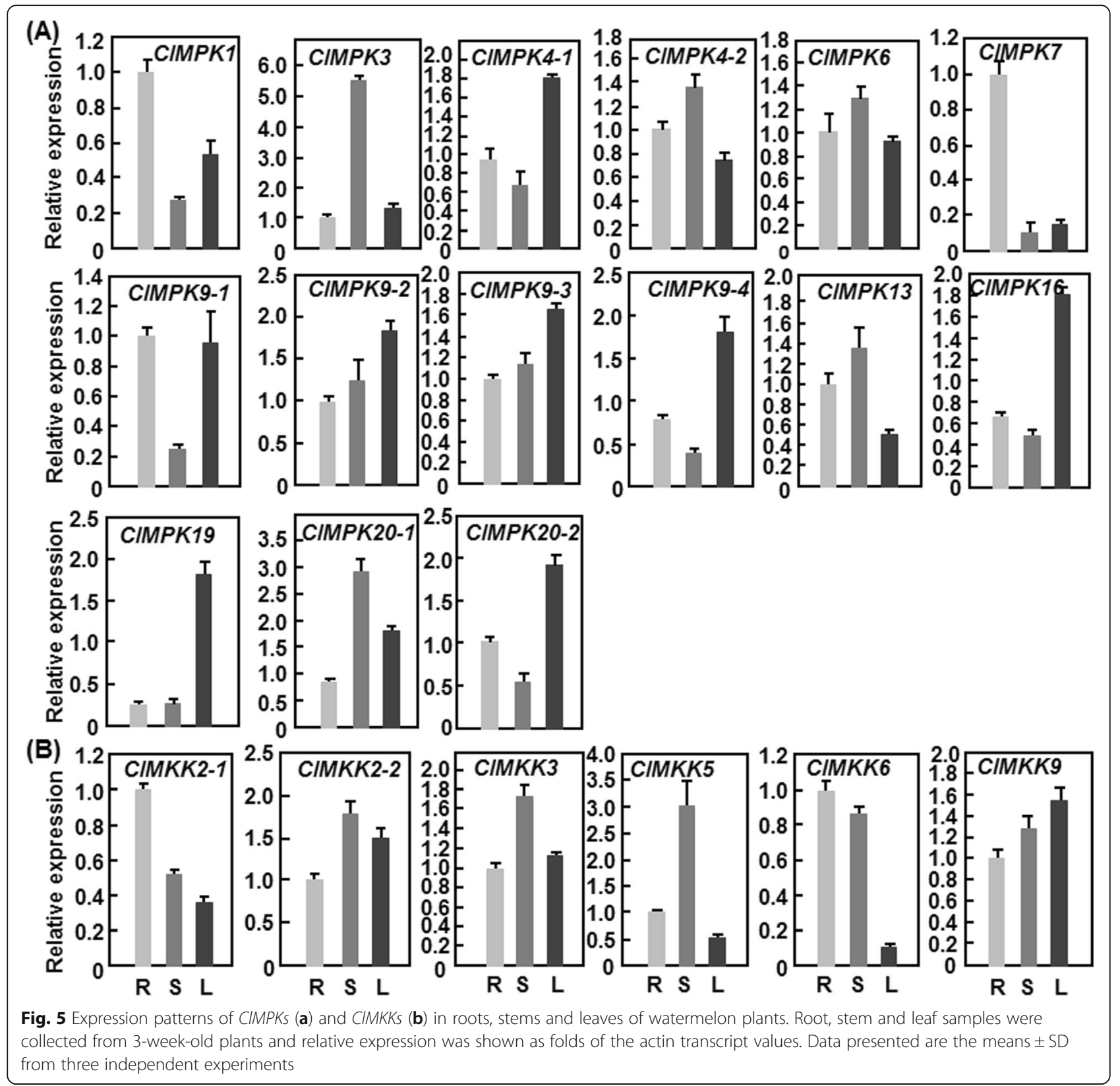

that the high levels of expression of these genes in specific tissues may be determined by their biological functions rather than the sequence similarity.

Expression patterns in response to abiotic stresses and $A B A$ It is well known that the MAPK cascades play important roles in abiotic stress responses in plants and some of the components of the MAPK cascades have been characterized as critical regulators of plant responses to drought, salt and temperature stresses [30, 31]. To explore the involvement of the ClMPK and ClMKK genes in abiotic stress responses, we analyzed by qRT-PCR their expression patterns and changes in expression in response to four stress treatments (drought, salinity, cold and heat) and to the stress hormone abscisic acid (ABA). Generally, the expression levels of $15 C l M P K$ and 6 $C l M K K$ genes were altered with distinct patterns in watermelon plants after treatment with drought, salinity, cold and heat stress and most of the ClMPK and ClMKK genes showed differential expression patterns in response to at least two treatments (Fig. 6). Specifically, 13 ClMPKs (ClMPK1, ClMPK3, ClMPK4-1, ClMPK4-2, ClMPК6, ClMPK7, ClMPK9-1, ClMPK9-2, ClMPK13, ClMPK16, ClMPK19, ClMPK20-1 and ClMPK20-2) and four ClMKKs (ClMKK2-1, ClMKK2-2, ClMKK3 and $C l M K K 5)$ were induced by drought stress (placing on 


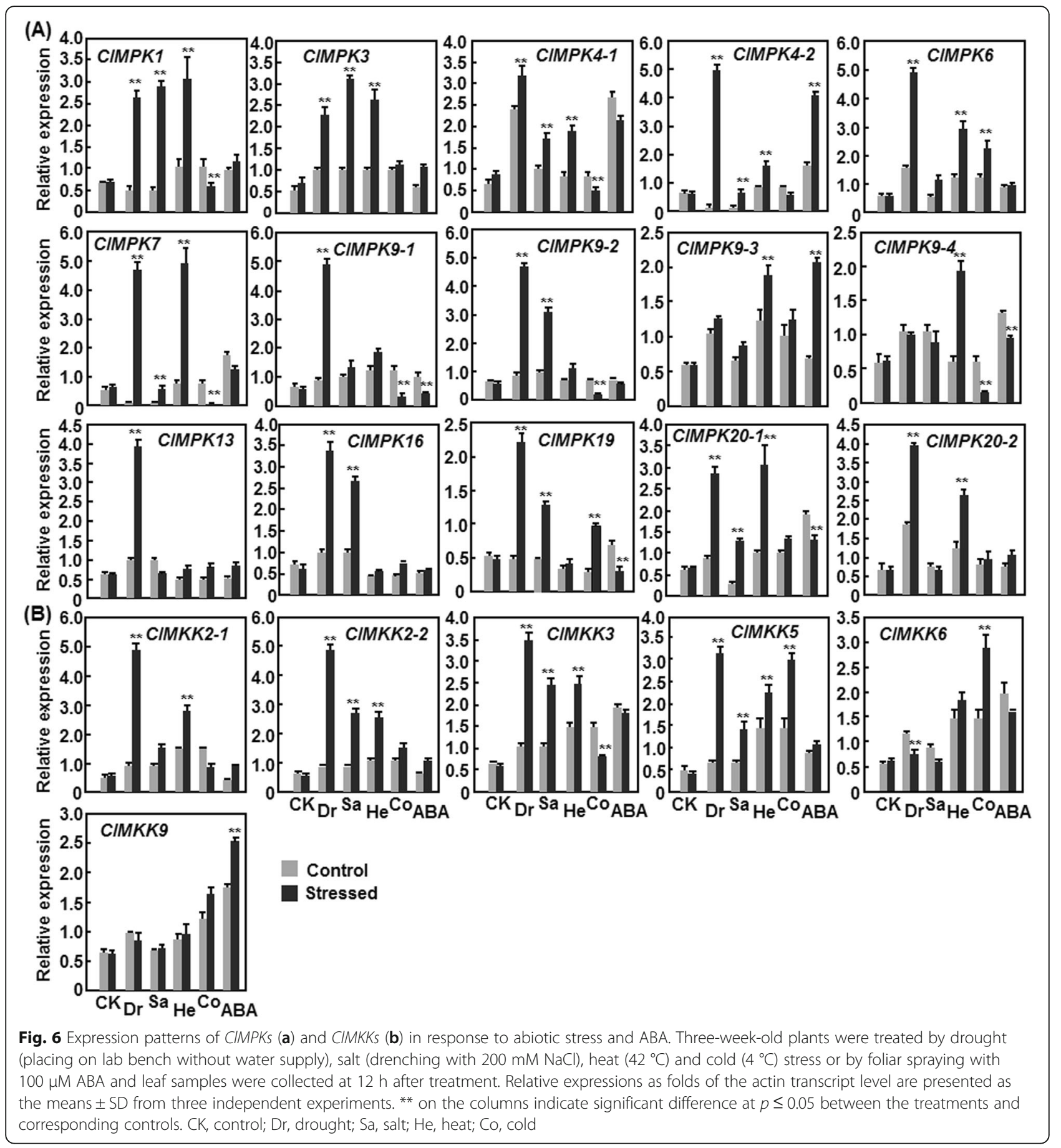

lab bench without water supply) (Fig. 6). Among them, the expression levels of ClMPK4-2 and ClMPK7 exhibited $>15$-fold increases at $12 \mathrm{~h}$ after drought stress treatment (Fig. 6a). In response to salt stress (drenching with $200 \mathrm{mM} \mathrm{NaCl}$ ), the expression of ten ClMPKs (ClMPK1, ClMPK3, ClMPK4-1, ClMPK4-2, ClMPK6, ClMPK7, ClMPK9-2, ClMPK16, ClMPK19 and ClMPK20-1) and three ClMKKs (ClMKK2-2, ClMKK3 and ClMKK5) was induced at different levels (Fig. 6). Under high temperature (heat treatment at $42{ }^{\circ} \mathrm{C}$ ) stress condition, the expression of ten ClMPKs (ClMPK1, ClMPK3, ClMPK4-1, ClMPK4-2, ClMPK6, ClMPK7, ClMPK9-3, ClMPK9-4, ClMPK20-2 and ClMPK20-2) and four ClMKKs (ClMKK2-1, ClMKK2-2, ClMKK3 and $C l M K K 5)$ was upregulated with different folds of increases over those in the control plants (Fig. 6). Among these heat-inducible ClMPK and ClMKK genes, the expression levels of ClMPK7, ClMPK9-4 
and ClMPK20-1 showed $>3$-fold of increases at $12 \mathrm{~h}$ after heat treatment (Fig. 6a). Unlike the upregulated expression patterns of most members in the ClMPK and $C l M K K$ families in response to drought, salt and heat stresses, the expression of ClMPKs and ClMKKs exhibited diverse patterns under low temperature condition (cold treatment at $4{ }^{\circ} \mathrm{C}$ ). For example, the expression levels of five ClMPKs (ClMPK6, ClMPK13, ClMPK16, ClMPK19 and ClMPK20-1) and three ClMKKs (ClMKK5, ClMKK6 and ClMKK9) were increased while the expression of seven ClMPKs (ClMPK1, ClMPK4-1, ClMPK4-2, ClMPK7, ClMPK9-1, ClMPK9-2 and ClMPK9-4) and two ClMKKs (ClMKK2-1 and ClMKK3) was downregulated at $12 \mathrm{~h}$ after cold treatment (Fig. 6). By contrast, the expression of ClMPK3, ClMPK9-3, ClMPK20-2 and ClMKK2-2 was not affected markedly under cold stress condition (Fig. 6). Collectively, some members such as ClMPK1, ClMPK3, ClMPK7 and ClMPK19 in the ClMPK family and ClMKK2-2, ClMKK3 and $C l M K K 5$ in the $C l M K K$ family exhibited upregulated expression under three stress treatments (Fig. 6), indicating that these ClMPK and ClMKK genes may have functions in response to multiple stresses. Interestingly, the expression of ClMPK7 was repressed in cold stress but was induced significantly in heat stress (Fig. 6a), suggesting that CIMPK7 may play opposite roles in cold and heat stress responses via different MAPK cascades. Furthermore, the expression of the paralog pair CIMPK4-1/ ClMPK4-2 showed similar patterns while the paralog pairs ClMPK9-1/ClMPK9-4, ClMPK20-1/ClMPK20-2 and ClMKK2-1/ClMKK2-2 exhibited distinct patterns in response to different abiotic stress treatments (Fig. 6).

It is well known that ABA and the ABA-mediated signaling pathway play central roles in abiotic stress response in plants through triggering major changes in gene expression and adaptive physiological responses $[30,32,33]$. Recently, MAPK cascades have been demonstrated to be implicated in ABA signaling that is involved in abiotic stress response [30]. Thus, we further analyzed the expression patterns of the ClMPK and ClMAKK genes in response to exogenous ABA. As shown in Fig. 6, the expression levels of three ClMPKs (ClMPK3, ClMPK4-2 and ClMPK9-3) and three ClMKKs (ClMKK2-1, ClMKK2-2 and ClMKK9) were increased while the expression levels of six ClMPKs (ClMPK4-1, ClMPK7, ClMPK9-1, ClMPK9-4, ClMPK19 and ClMPK20-1) and one ClMKK (ClMKK6) were decreased after ABA treatment. By contrast, the expression of ClMPK1, ClMPK6, ClMPK9-2, ClMPK13, ClMPK16, ClMPK20-2, ClMKK3 and ClMKK5 was not affected by exogenous ABA (Fig. 6). Notably, the expression of some members such as ClMPK4-1, ClMPK7, ClMPK19 and ClMPAK20-1 in the ClMPK family and ClMKK3 in the $C l M K K$ family showed distinct and even opposite patterns in response to abiotic stress and exogenous ABA (Fig. 6). This does not imply that ABA and its signaling are not involved in the response to abiotic stresses that regulate the expression of these ClMPKs and $C l M K K s$ as the activity and function of the MAPK cascades depend largely on the phosphorylation status of the components.

\section{Expression patterns in response to pathogen infection}

The functions of MAPK cascades in plants disease resistance have been well documented both in the model plants and crops $[1,6]$. To explore the involvement of ClMPKs and ClMKKs in disease resistance, we analyzed their expression patterns in watermelon plants after infection with Fusarium oxypsorum f. sp. niveum (Fon), the most important soilborne fungal pathogen causing Fusarium wilt disease limiting watermelon production in many areas of the world $[34,35]$. To do this, we inoculated the two-week-old plants with Fon spore suspension and monitored the disease progress over a period of 3 weeks. In our 4 independent experiments, the average of the disease incidence was approximately $90 \%$. Typical symptom of Fusarium wilt disease, showing wilted leaves, was observed at 9 days after inoculation (dpi) in Fon-inoculated plants but not in the mock-inoculated plants and most of the Fon-inoculated plants died at 18 dpi (Fig. 7a). To examine the defense response in watermelon plants after infection by Fon, we analyzed and compared the expression patterns of two defense-related genes, ClPR5 and Chitinase, in the Fon-inoculated and mock-inoculated plants. As shown in Fig. 7b, the expression levels of ClPR5 and Chitinase in the Fon-inoculated plants were comparable to those in the mock-inoculated plants at $6 \mathrm{dpi}$; however, the levels in the Fon-inoculated plants were significantly increased at $9 \mathrm{dpi}$, showing approximately 8 - and 3 -fold of increases over those in the mock-inoculated plants (Fig. 7b), indicating an activation of defense response in the Fon-inoculated plants. We then analyzed the expression patterns of ClMPKs and ClMKKs in response to Fon using the samples collected form the Fon- and mock-inoculated plants, which were verified by monitoring of disease progress and expression of defense-related genes (Fig. $7 \mathrm{a}$ and $\mathrm{b}$ ). As shown in Fig. 7c and d, the expression levels of 12 ClMPKs (ClMPK1, ClMPK3, ClMPK4-1, ClMPK4-2, ClMPK6, ClMPK7, ClMPK9-1, ClMPK9-2, ClMPK9-3, ClMPK13, ClMPK16 and ClMPK20-1) and four ClMKKs (ClMKK21, ClMKK2-2, ClMKK5 and ClMKK6) were altered with distinct patterns in watermelon plants after Fon infection, indicating that these ClMPKs and ClMKKs are Fon-inducible. However, the expression of ClMPK9-4, ClMPK19, ClMPK20-2, ClMKK3 and ClMKK9 was not affected significantly by Fon infection. Furthermore, the expression of these Fon-inducible ClMPKs and ClMKKs 


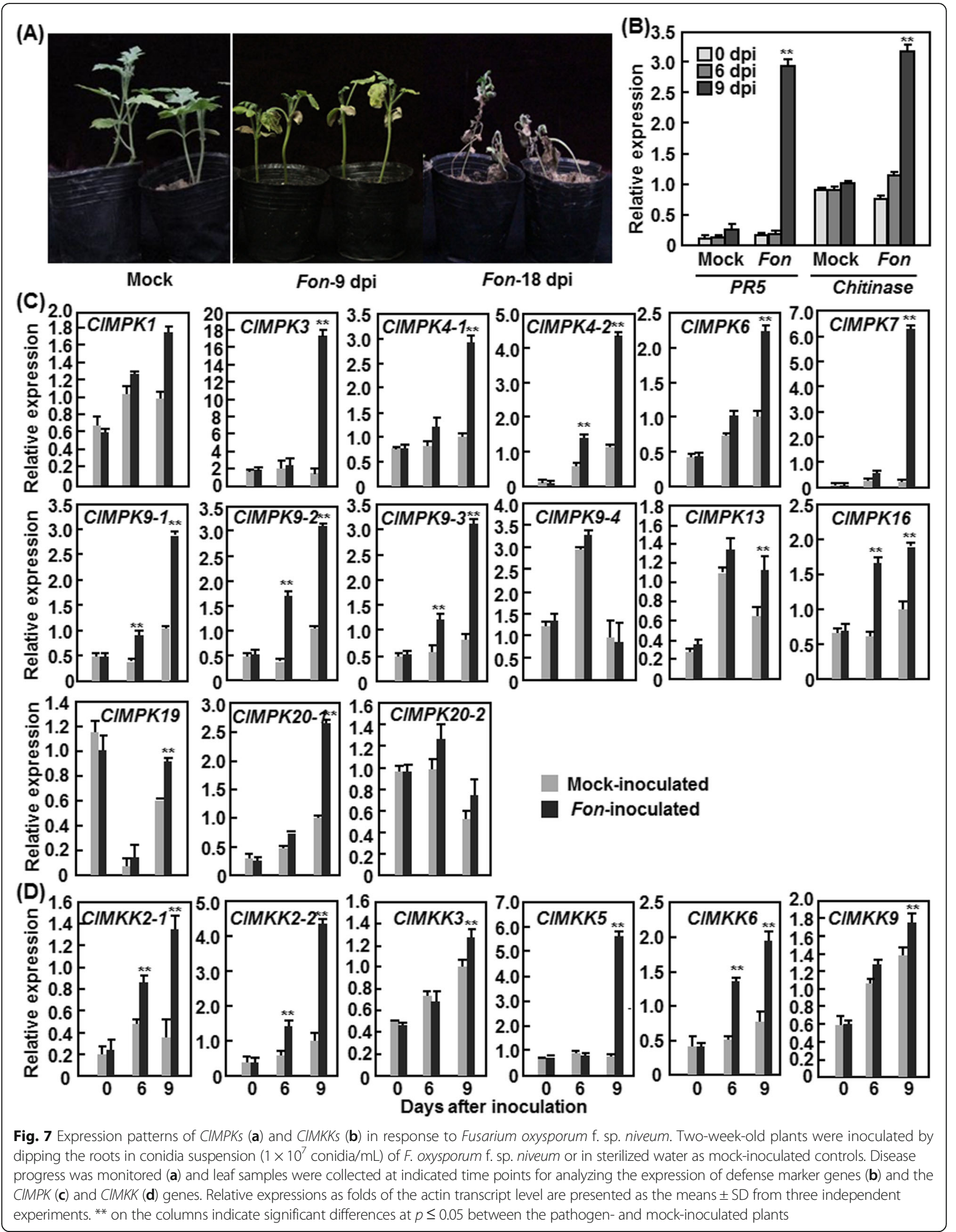


exhibited distinct patterns in terms of time-course and magnitude of the Fon-induced expression. For example, the expression levels of ClMPK9-2, ClMPK16 and ClMKK6 were increased significantly at $6 \mathrm{dpi}$ and showed further increases at $9 \mathrm{dpi}$ while the expression levels of other Fon-inducible ClMPKs and ClMKKs were only increased significantly at 9 dpi (Fig. $7 \mathrm{c}$ and d). At 9 dpi, the expression levels of ClMPK2-1, ClMPK2-2, ClMPK4-1, ClMPK4-2 and ClMPK9-3 exhibited $>3$-fold and the expression levels of ClMPK3, ClMPK7 and ClMKK5 showed $>6$-fold of increases over those in the mock-inoculated plants (Fig. 7c and d).

\section{Functions of CIMPK1, CIMPK4-2, CIMPK7, CIMPK6 and CIMKK2-2 in disease resistance}

Due to the unavailability of routine transformation of watermelon, we therefore performed functional analyses through ectopic transient expression in $N$. benthamiana to further investigate the functions of ClMPKs and ClMKKs in disease resistance. To this end, 9 ClMPKs (ClMPK1, ClMPK3, ClMPK4-1, ClMPK4-2, ClMPK6, ClMPK7, ClMPK9-2, ClMPK13 and ClMPK19) and 5 ClMKKs (ClMKK2-1, ClMKK2-2, ClMKK5 and ClMKK6 and $C l M K K 9)$ were transiently expressed in $N$. benthamiana via agroinfiltration. qRT-PCR analysis with samples collected at $24 \mathrm{~h}$ after agroinfiltration indicated that most of the selected ClMPKs and ClMKKs expressed normally in $N$. benthiamina and their expression levels, shown as folds of the level of a NbActin gene, varied greatly in individual ClMPK- or ClMKK-infiltrated leaves while no transcript for the selected ClMPKs and ClMKKs was detected in eGFP-infiltrated leaves (Fig. 8a). The expression levels of ClMPK7, ClMPK1 and ClMKK2-2 were approximately 16-, 5.5- and 3.4-fold, and the expression levels of the remaining selected ClMPKs and ClMKKs were about 0.3-2.1-fold of the NbActin gene (Fig. 8a). Unfortunately, we were unable to detect the expression of ClMPK9-2 and ClMKK9 in N. benthamiana and thus we did not perform further experiments on these two genes. At $48 \mathrm{~h}$ after agroinfiltration for transient expression, the agroinfiltrated leaves were collected for disease assays by dropping spore suspension of $B$. cinerea on both sides of the leaves. Disease phenotyping at 3 day after inoculation revealed that the B. cinerea-caused lesions on ClMPK3-, ClMPK4-1-, ClMPK13-, ClMPK19-, ClMKK2-1-, ClMKK5- or ClMK K6-infiltrated leaves were comparable to those on eGFPor buffer-infiltrated control leaves (Fig. $8 \mathrm{~b}$ and c), suggesting that transient expression of these ClMPKs and ClMKKs in N. benthamiana did not affect the resistance to $B$. cinerea. By contrast, the $B$. cinerea-caused lesions on ClMPK1-, ClMPK4-2-, and ClMPK7-infiltrated leaves were significantly smaller (Fig. 8b), showing 38,36 and $80 \%$ of decrease in size, respectively (Fig. 8c), while the lesions on ClMPK6- and ClMKK2-2-infiltrated leaves were markedly larger (Fig. 8b), leading to 103 and $87 \%$ of increase in size, respectively (Fig. 8c), as compared with those on eGFP- or buffer-infiltrated leaves, indicating that transient expression of ClMPK7, ClMPK1, ClMPK4-2, ClMPK6 or ClMKK2-2 in N. benthamiana affected the resistance to $B$. cinerea. Analysis of the transcript for the $B$. cinerea actin gene BcActinA as an indicator of the rate of in planta fungal growth indicated that growth of B. cinerea in the ClMPK1-, ClMPK4-2-, and ClMPK7-infiltrated leaves was significantly lower, showing 52, 50 and $91 \%$ of decrease, respectively; whereas the growth in the ClMPK6- and ClMKK2-2-infiltrated leaves was markedly higher, resulting in 72 and $160 \%$ of increase, respectively, as compared with those on eGFP-infiltrated control leaves (Fig. 8d).

It was previously shown that overexpression of the Arabidopsis AtMKK7 leads to activation of systemic acquired resistance [36], a form of inducible immune responses in plants [37]. We therefore examined whether transient expression of ClMPK1, ClMPK4-2, ClMPK7, ClMPK6 or ClMKK2-2 in N. benthamiana affect the resistance of distal tissues to B. cinerea. For this purpose, agrobacteria carrying the constructs containing ClMPK1, ClMPK4-2, ClMPK7, ClMPK6 or ClMKK2-2 were infiltrated into one half of the leaves and disease assays with $B$. cinerea were performed on the opposite half of the agroinfiltrated leaves at 2 days after agroinfiltration. Disease phenotyping at 3 day after inoculation revealed that the $B$. cinerea-caused lesions on the opposite half of the ClMPK1-, ClMPK4-2-, and ClMPK7-infiltrated leaves were significantly smaller (Fig. 9a), showing 38, 25 and $64 \%$ of decrease in size, respectively (Fig. 9b), while the lesions on the opposite half of the ClMPK6- and ClMKK2-2-infiltrated leaves were markedly larger (Fig. 9a), resulting in 12 and $35 \%$ of increase in size, respectively (Fig. 9b), as compared with those on eGFPinfiltrated control leaves.

To explore the possible molecular mechanisms for the actions of ClMPK1, ClMPK4-2, ClMPK7, ClMPK6 and ClMKK2-2 in disease resistance, we analyzed whether transient expression of these ClMPKs and ClMKKs affected the expression of defense-related genes in $N$. benthamiana. The expression levels of NbPR1, NbPR2 and NbPR5, three defense-related genes [38], in ClM PK1-, ClMPK4-2-, ClMPK7-, ClMPK6- or ClMKK2-2transiently expressed leaves were analyzed and compared with those in eGFP-infiltrated leaves. As shown in Fig. 9c, no expression of the tested defense-related genes was detected at $0 \mathrm{~h}$ after agroinfiltration; however, increased expression of these genes at $24 \mathrm{~h}$ after agroinfiltration in ClMPK1-, ClMPK4-2-, ClMPK7-, or ClMKK2-2-transiently expressed leaves was observed. The expression levels of NbPR1 and NbPR5 were significantly increased 


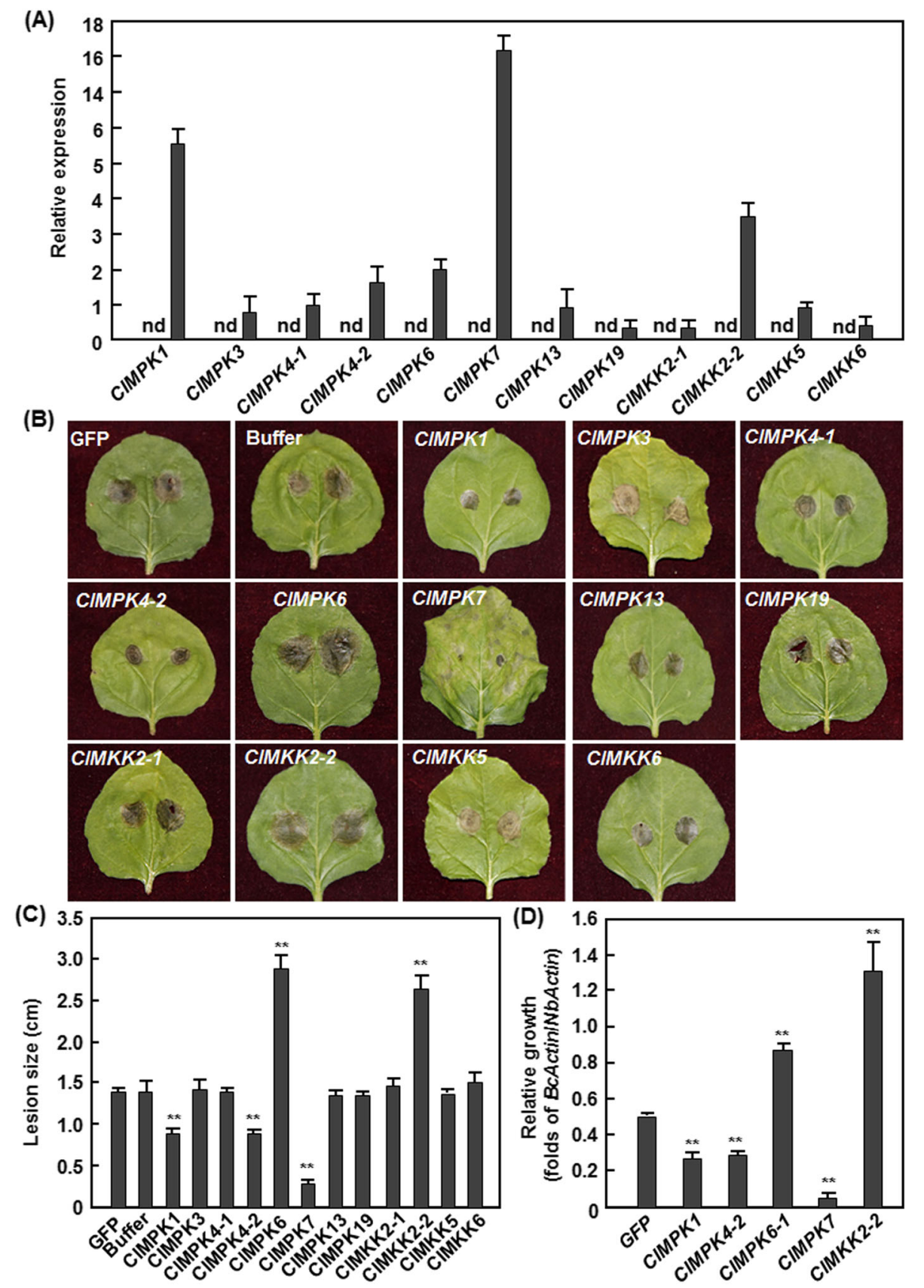

Fig. 8 Disease phenotype in CIMPK- and CIMKK-transiently expressed N. benthamiana leaves after inoculation with B. cinerea. Agrobacteria harboring different constructs containing CIMPKs, CIMKKs or eGFP (a negative control) or similar volume of buffer (a negative control) were infiltrated into leaves of 4-week-old N. benthamiana plants and the agroinfiltrated leaves were collected for analyzing the expression of CIMPKS and CIMKKs and for disease assays with B. cinerea. a Expression levels of selected CIMPKs and CIMKKs in agroinfiltrated leaves. Leaf sample were collected at $24 \mathrm{~h}$ after agroinfiltration and relative expressions as folds of the actin transcript level are presented as the means \pm SD from three independent experiments. nd, expression of the CIMPKs and CIMKKs in eGFP-infiltrated leaves was not detectable. b Disease phenotype and c lesion sizes on detached leaves and $\mathbf{d}$ fungal growth in the inoculated leaves. The agroinfiltrated leaves were detached at 2 days after agroinfiltration and disease assays were performed by dropping $5 \mu \mathrm{L}$ of spore suspension $\left(1 \times 10^{5}\right.$ spores $\left./ \mathrm{mL}\right)$. Photos were taken and lesion sizes were recorded at 4 days after inoculation. Fungal growth in inoculated leaves was assumed by analyzing the transcripts of BcActin gene by qRTPCR using NbActin as an internal control at 4 days after inoculation. Data presented in $\mathbf{c}$ and $\mathbf{d}$ are the means \pm SD from three independent experiments and ** on the columns indicate significant difference at $p \leq 0.05$ between CIMPK/CIMKK- and eGFP-infiltrated plants 


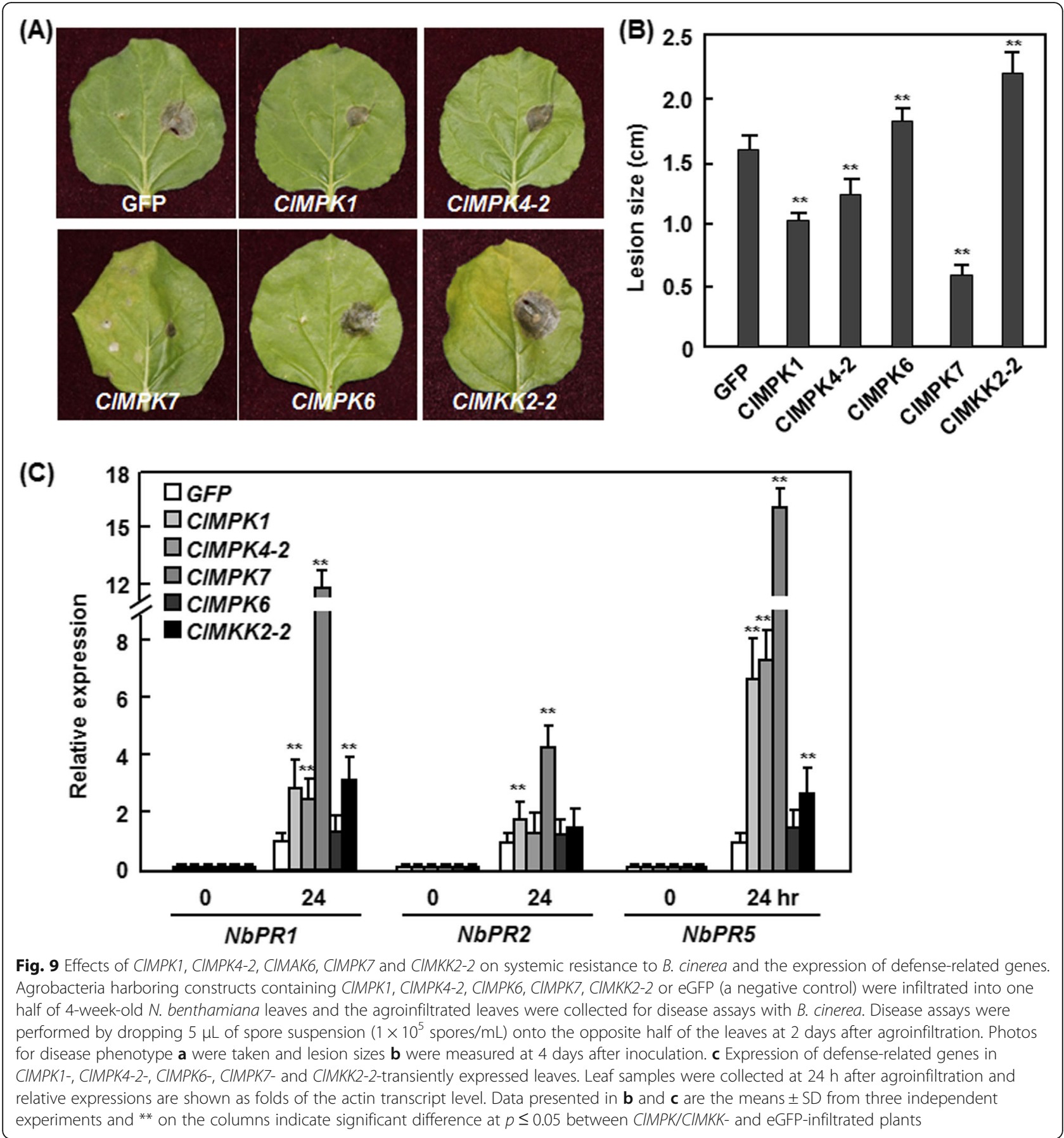

at $24 \mathrm{~h}$ after agroinfiltration in ClMPK1-, ClMPK4-2- or ClMPK7-transiently expressed leaves, leading to 1.3 10.8 folds for NbPR1 and $5.5 \sim 15.5$ folds for NbPR5 over those in the eGFP-infiltrated leaves (Fig. 9c). Increased expression of NbPR2 in ClMPK1- or ClMPK7-transiently expressed leaves and of NbPR1 and NbPR5 in ClMKK22-transiently expressed leaves were also observed (Fig. 9c). However, the expression levels of NbPR1, NbPR2 and NbPR5 in ClMPK6-transiently expressed leaves were comparable to those in eGFP-infiltrated leaves (Fig. 9c).

Function of CIMPK7 in hypersensitive response-like cell death

During our transient expression-based functional analysis of the selected ClMPKs and ClMPKs in disease resistance, we noted that the ClMPK7-transiently expressed leaves exhibited typical hypersensitive response (HR)-like cell 
death while other selected ClMPKs or ClMKKs-transiently expressed leaves did not (Fig. 9a), indicating an involvement of ClMPK7 in HR-like cell death. Therefore, several experiments were conducted to confirm the possible function of ClMPK7 in HR-like cell death. At $24 \mathrm{~h}$ after agroinfiltration, significant accumulation of the ClMPK7 protein as a ClMPK7-GFP fusion was clearly detected in
ClMPK7-GFP-infiltrated leaves while only GFP was detected in eGFP-infiltrated leaves (Fig. 10a). In ClMPK7GFP-infiltrated leaves, typical HR-like cell death as small necrotic lesions in the infiltration area was observed at $24 \mathrm{~h}$ and these necrotic lesions enlarged with times, forming large necrotic area at 7 days after agroinfiltration (Fig. 10b). Only slight cell death at the infiltration site was

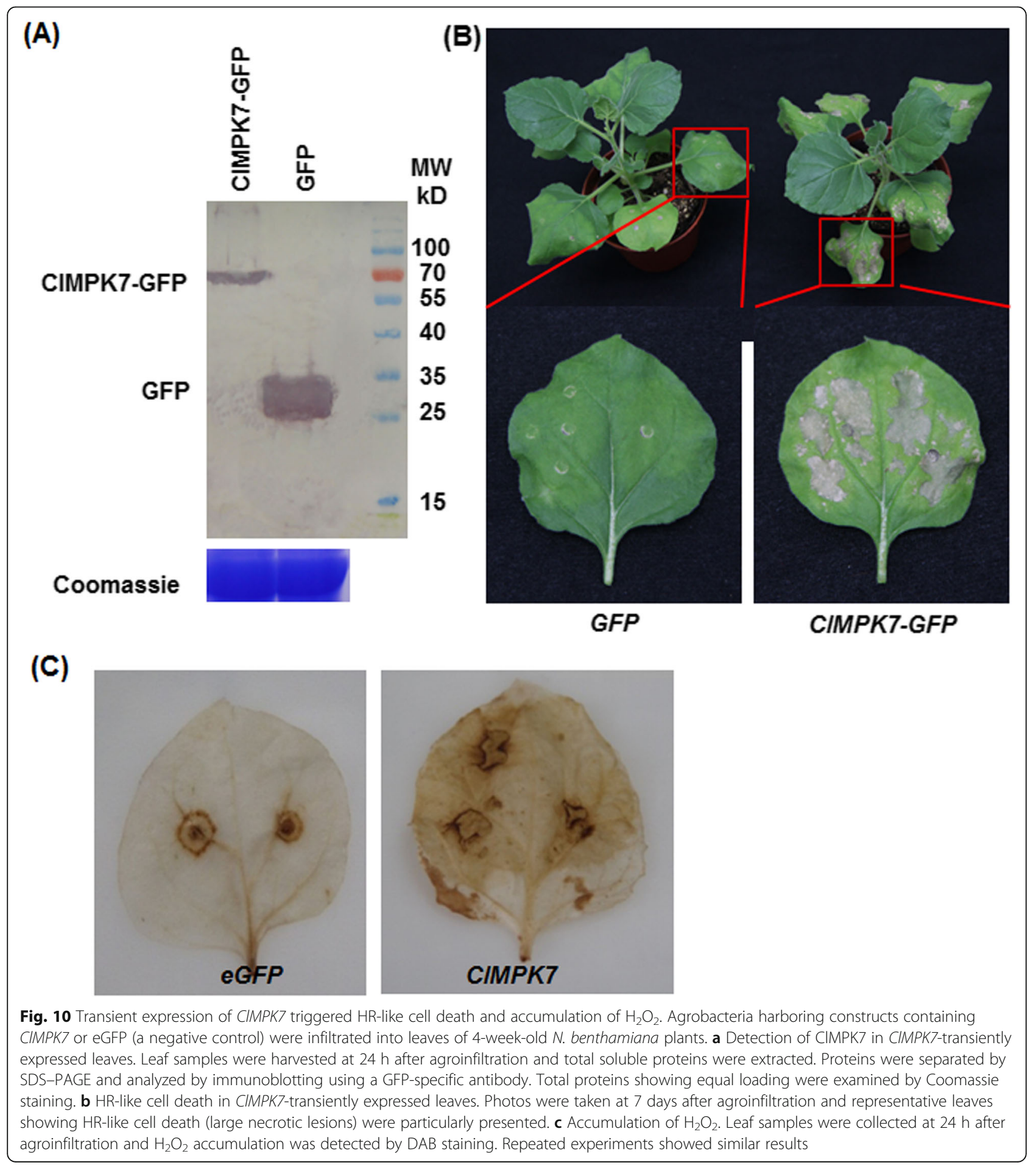


observed in eGFP-infiltrated leaves, probably due to wounding during infiltration process (Fig. 10b). Furthermore, significant accumulation of $\mathrm{H}_{2} \mathrm{O}_{2}$, as detected by 3 , 3-diaminobenzidine (DAB) staining, was observed in ClMPK7-GFP-infiltrated leaves, not only at the infiltration site but also in the tissues surrounding the infiltration sites, while the $\mathrm{H}_{2} \mathrm{O}_{2}$ accumulation was only seen at the infiltration sites (Fig. 10c). These data indicate that ClMPK7 plays a role in HR-like cell death probably through modulating the generation of $\mathrm{H}_{2} \mathrm{O}_{2}$.

\section{Discussion}

The MAPK cascades are one of the major pathways that play critical roles in growth and development as well as in stress responses. The MPKs and MKKs, the two last components in the MAPK cascades, are represented as multigene families, which have been studied in detail at the genome-wide level in a number of plants species. Our genome-wide survey identified $15 \mathrm{ClMPKs}$ and 6 ClMKKs in watermelon, which can be classified into four distinct groups. Data from our detailed studies on some selected members of the ClMPK and ClMKK families for their protein-protein interaction relationships, expression patterns in different tissues and in response to abiotic and biotic stress, and possible functions in disease resistance provide the first line of evidence for the biological functions of the CIMPK and CIMKK families in watermelon.

The function and activity of components in the MAPK cascades depend on their direct physical interactions. In the present study, complicated interaction relationships and specificity between ClMPKs and ClMKKs were observed. For examples, CIMPK4-1 interacted with two ClMKKs (ClMKK2-1 and ClMKK6) while ClMKK2-1 interacted with three ClMPKs (ClMPK4-1, ClMPK4-2 and ClMPK13 (Fig. 4). ClMKK2-2 interacted specifically with ClMPK1 and vice versa (Fig. 4). ClMKK2-1 and ClMKK2-2, which have high levels of sequence similarity (Fig. 2), interacted with different ClMPKs (Fig. 4). The complicated interaction relationships and specificity between ClMPKs and ClMKKs indicate that they may integrate into divergent signaling pathways and determine specific biological functions [2, 39].

The CD domain, which is thought to be involved in interacting with upstream MKKs [40], seems not the sole domain responsible for protein-protein interaction between ClMPKs and ClMKKs. It is reasonable that ClMPK4-1, ClMPK4-2, CIMPK6 and ClMPK13 interacted differentially with corresponding ClMKKs (Fig. 4), as all these four ClMPKs contain the CD domain. Surprisingly, however, CIMPK1 and CIMPK7, which do not have the CD domain, interacted with CIMKK2-2 and ClMKK5, respectively (Fig. 4). This is similar to the previous observations that $B$. distachyon BdMPK7-1/14/17 and canola BnaMPK9/19/20, all lacking the CD domain, could interact with upstream corresponding MKKs [17, 22]. Thus, it is likely that other domains/motifs in ClMPKs may be involved in determination of the interaction with upstream ClMKKs.

It was previously demonstrated that the Arabidopsis AtMKK1/AtMKK2 interact with AtMPK4, forming AtMKK1/AtMKK2-AtMPK4 cascade, while AtMKK4/ AtMKK5 interact with both of AtMPK3/AtMPK6, leading to AtMKK4/AtMKK5-AtMPK3/AtMPK6 cascades [41-45]. Similar interactions between ClMPKs and ClMKKs were observed. For example, ClMKK2-1, closely related to AtMKK1/AtMKK2 (Fig. 2), interacted significantly with ClMPK4-1 and CIMPK4-2, two CIMPKs that are phylogenetically clustered with AtMPK4 (Fig. 2), whereas CIMKK5, a putative ortholog of AtMKK4 and AtMKK5, interacted strongly with ClMPK6, a ClMPK with high level of similarity to AtMPK6. The fact that ClMKK6 interacted with ClMPK2-1, ClMPK2-2 and ClMPK13 is similar to the Arabidopsis AtMKK6, which can interact and phosphorylate AtMPK4 and AtMPK13 [46-48]. Interestingly, ClMKK2-1 and ClMKK6 interacted with the same ClMPKs including ClMPK4-1, ClMPK4-2 and ClMPK13 (Fig. 4). Collectively, it is likely that ClMKK2-1/ClMKK6-ClMPK4-1/ClMPK4-2/ClMPK13 and ClMKK5-ClMPK6 in watermelon may constitute separate MAPK cascades. However, like those in Arabidopsis and rice [43, 49], further comprehensive analysis of protein-protein interactions among ClMKKs and ClMPKs will be helpful to establish the MAPK cascades and their signaling networks.

Although activity of the MAPK cascades can be regulated at both transcriptional and post-translational levels, transcriptional regulation of expression of MPK and MKK genes was reported previously in a range of plants. It is generally accepted that a gene expressed abundantly in a tissue or during a developmental stage or increasingly under a stress condition may imply its function related to developmental and stress response. In this regard, the expression patterns of ClMPKs and ClMKKs in different tissues or in response to biotic and abiotic stresses may indicate the biological functions of and the possible relationships between ClMPKs and ClMKKs in watermelon. The expression of members in the putative ClMKK2-1/ClMKK6-ClMPK4-1/ClMPK4-2/CIMPK13 and CIMKK5-CIMPK6 cascades showed similar upregulated patterns in response to Fon infection (Fig. 7). Similarly, the expression of ClMPK6 and ClMKK5 in the ClMKK5-CIMPK6 cascade was synchronously upregulated by drought, heat and cold stresses (Fig. 6). However, different expression patterns of the members in these two putative MAPK cascades in different tissues and upon abiotic and biotic stress treatments were also noted. For example, salt stress induced the expression of 
ClMPK4-1 and ClMPK4-2 but did not affect the expression of ClMKK2-1 and ClMKK6 (Fig. 6). The difference in expression patterns of the members in a putative MPAK cascade may be explained by the nature that the biochemical function of the MAPK cascades is mainly determined by the phosphorylation status of the components in the cascades or that other components exist to form unknown cascades under specific growth and stress conditions. Another, different expression patterns of some paralog pairs were observed. For example, the expression of the paralog pair CIMPK4-1/ClMPK4-2 showed similar patterns while the paralog pairs CIMPK9-1/ClMPK9-4, ClMPK20-1/ClMPK20-2 and ClMKK2-1/ClMKK2-2 exhibited distinct expression patterns in response to different abiotic stress treatments (Fig. 6). This is similar to the results observed in the cotton MPK family under different abiotic stress treatments [20]. It is thus likely that some members of the CIMPK and ClMKK families may retain the functional conservation while others evolve to possess divergent functions to cope with different environmental challenges.

Our expression analyses revealed that the CIMPK and $C l M K K$ families respond with different patterns to Fon infection and that ClMPK3, ClMPK7 and ClMKK5 were significantly induced by Fon (Fig. 7), indicating their possible involvements in the activation of defense response in watermelon to Fon. Further transient expression-based functional analyses demonstrated that ClMPK1, ClMPK42 and ClMPK7 positively but ClMPK6 and ClMKK2-2 negatively regulate the resistance to $B$. cinerea when transiently expressed in $N$. benthamiana (Fig. 8). The fact that transient expression of ClMPK1, ClMPK4-2, ClMPK7, ClMPK6 and ClMKK2-2 in N. benthamiana affected the resistance of distal tissues to $B$. cinerea not only confirmed their functions in disease resistance but also suggest systemic effects on activation of defense response (Fig. 9). ClMPK1 and ClMPK7 belong to group $\mathrm{C}$ and phylogenetically related to Arabidopsis AtMPK1, AtMPK2, AtMPK7 and AtMPK14 (Fig. 2). In the present study, we found that the expression of $C l M P K 7$ was induced by several abiotic stresses and by Fon (Figs. 6 and 7) and transient expression of ClMPK7 in $N$. benthamiana resulted in increased resistance to B. cinerea (Figs. 8 and 9). This is consistent with the observations that the Arabidopsis AtMPK7, as a component of the AtMKK3-AtMPK7 cascade, was found to play a role in defense responses against $P$. syringae pv. tomato DC3000 while overexpression of cotton GhMPK7 in $N$. benthamiana conferred an increased resistance to Colletotrichum nicotianae [50, 51]. ClMPK4-2 is closely related to Arabidopsis AtMPK4 (Fig. 2a). Expression of ClMPK4-2 was induced at $6 \mathrm{dpi}$ after infection of Fon (Fig. 7) and transient expression in $N$. benthamiana resulted in increased resistance to B. cinerea (Figs. 8 and 9). It was previously shown that the Arabidopsis atmpk4 mutant and tomato SlMPK4-silenced plants showed enhanced susceptibility to Alternaria brassicicola and $B$. cinerea, respectively $[52,53]$, whereas overexpression of $B n M P K 4$ in oilseed rape plants significantly enhances resistance to Sclerotinia sclerotiorum and B. cinerea [54]. These data demonstrate that plant MPK4 including ClMPK4-2 functions as positive regulators of defense response against necrotrophic fungal pathogens. Another ClMPK that has function in disease resistance to $B$. cinerea is CIMPK6, showing high level of similarity to Arabidopsis AtMPK6 (Fig. 2a), which is well documented as a critical component of the MEKK1-MKK4/MKK5MPK3/MPK6 cascades regulating immune responses $[1$, 6 , 7]. It was found that activation of AtMPK3 and AtMPK6 impeded the infection of $B$. cinerea [55] although lack of AtMPK6 did not affect the basal resistance to $B$. cinerea [56]. This is somewhat different from our observation in the present study that transient expression of ClMPK6 in N. benthamiana led to reduced resistance to B. cinerea (Figs. 8 and 9). ClMKK2-2 is a putative ortholog of Arabidopsis AtMKK1 and AtMKK2 (Fig. 2b) in the AtMKK1/AtMKK2-AtMPK4 cascade, which negatively regulates immunity [45]. However, ClMKK2-2 did not interact with ClMPK4-2 (Fig. 4b), which is closely related to AtMPK4 and AtMPK11, and its function of ClMKK2-2 in resistance to $B$. cinerea differs from that of ClMPK4-2 (Figs. 8 and 9). Thus, it is unlikely that ClMKK2-2 and ClMPK4-2 form a functional MAPK cascade. Although ClMKK2-2 interacted with ClMPK1 (Fig. 4b), they had opposite effects on the resistance to $B$. cinerea when transiently expressed in N. benthamiana (Figs. 8 and 9). Whether ClMKK2-2 and ClMPK1 form a true functional MAPK cascade needs to be further examined.

We also found in the present study that transient expression of ClMPK7 in $N$. benthamiana triggered a HRlike cell death and that ClMPK7-induced HR-like cell death was probably initiated by abnormal ROS accumulation (Fig. 10). This is similar to the previous observations that activation of the tobacco SIPK/Ntf4/WIPK and the Aabidopsis AtMKK4/AtMKK5 cascades actively promotes the generation of ROS, which plays an important role in the signaling for and/or execution of HR cell death [57-59]. Notably, transient expression of ClMPK7 in $N$. benthamiana resulted in significant HR-like cell death and increased resistance to $B$. cinerea (Figs. 8, 9 and 10), which is consistent with the functions of Arabidopsis AtMKK4, tobacco NtMEK2 and tomato SIMKK2/ SIMKK4 in HR-like cell death and enhanced resistance to $B$. cinerea $[16,41,58]$. On the other hand, it is well known that expression of constitutively active forms of MKKs can trigger HR-like cell death in plants [16, 38, $41,58]$. However, we did not observed any HR-like cell death in leaves of $N$. benthamiana plants infiltrated 
constructs carrying wild type forms of ClMKK2-1, ClMKK2-2, ClMKK5 and ClMKK6 (Fig. 8), indicating that transient expression of the wild type forms of these ClMKKs cannot trigger HR-like cell death. This is consistent with the observations that overexpression of wild type forms of tomato SIMKK2 and SIMMK4 and the Arabidopsis AtMKK3 did not induce HR-like cell death or affect disease resistance but overexpression of the constitutively active phosphomimicking forms induced significant HR-like cell death or disease resistance $[16,50]$. Therefore, the functions of ClMKK2-1, ClMKK2-2, ClMKK5 and ClMKK6 in HR-like cell death need to be further investigated using the constitutively active phosphomimicking forms.

\section{Conclusion}

To date, little is known about the MPK and MKK families and their possible biological functions in watermelon. In addition to the genome-wide characterization of the ClMPK and CIMKK families in watermelon, the present study demonstrated significant interactions between members of the ClMPK and ClMKK families including putative CIMKK2-1/CIMKK6-ClMPK4-1/ClMP K4-2/ClMPK13 and ClMKK5-ClMPK6 cascades and showed the differential expression patterns for most of the members in the ClMPK and ClMKK families in different tissues and in response to abiotic (e.g. drought, salt, cold and heat treatments) and biotic (e.g. Fon infection) stresses. Importantly, we found that ClMPK1 and ClMPK7 in Group C and ClMPK4-2 in Group B positively but ClMPK6 in Group A and ClMKK2-2 in Group A of ClMKKs negatively regulate the resistance to $B$. cinerea when transiently expressed in $N$. benthamiana and that CIMPK7 in Group C functions as a regulator of HR-like cell death. The expression patterns, proteinprotein interaction relationship, possible functions in disease resistance and their potential functional Arabidopsis orthologs of the CIMPK and CIMKK families are summarized in Table 2. The present work provides an important foundation to direct future functional studies of the ClMPK and ClMKK families in growth/development and stress responses in watermelon. Further genetic studies in watermelon through overexpression and RNA interference approaches will be critical to elucidate the biological functions and molecular mechanisms of the ClMPKs and ClMKKs.

\section{Methods}

\section{Plant growth and treatments}

Watermelon (Citrullus lanatus) cv. Zaojia was used for all experiments. Plants were grown in a mixture of perlite: vermiculite: plant ash (1:6:2) in a growth room under fluorescent light $\left(200 \mu \mathrm{E} \mathrm{m} \mathrm{s}^{-1}\right)$ at $22-24{ }^{\circ} \mathrm{C}$ with $60 \%$ relative humidity and a $14 \mathrm{~h}$ light $/ 10 \mathrm{~h}$ dark cycle and three-week-old plants were used unless indicated otherwise. For analysis of tissue-specific expression, leaf, stem and root samples were collected and stored at $-80{ }^{\circ} \mathrm{C}$ till use. For ABA treatment, plants were treated by spraying with $100 \mu \mathrm{M} \mathrm{ABA}$ or with equal volume of solution containing only $0.1 \%$ ethanol and $0.02 \%$ Tween-20 as a control. For cold stress treatment, plants were transferred to a growth chamber at $4{ }^{\circ} \mathrm{C}$ or kept at $25{ }^{\circ} \mathrm{C}$ as a control for $24 \mathrm{~h}$. For heat treatment, plants were transferred to a growth chamber at $42{ }^{\circ} \mathrm{C}$ or kept at $25{ }^{\circ} \mathrm{C}$ as a control for $24 \mathrm{~h}$. For drought stress treatment, plants were put on lab blench without water supply or on water-saturated filter papers as a control for $12 \mathrm{~h}$. For salt stress treatment, plants were irrigated with $200 \mathrm{mM} \mathrm{NaCl}$ solution or water as a control at $25{ }^{\circ} \mathrm{C}$. For analysis of gene expression in response to Fon infection, inoculation was performed according to a previously reported method [60]. Briefly, conidia were collected from 10-day-old culture of Fon race 1 and adjusted to $1 \times 10^{6}$ conidia/mL. Two-week-old plants were carefully uprooted, washed in tap water and then roots of the plants were dipped for $30 \mathrm{~s}$ in the conidial suspension or in distilled sterilized water as mock-inoculated controls. The inoculated plants were carefully replanted in soil and allowed to grow in the same growth room as described above. Leaf samples were collected at indicated time points after the treatments and stored at $-80{ }^{\circ} \mathrm{C}$ till use.

\section{Characterization and nomenclature of the watermelon CIMPK and CIMKK genes}

The Arabidopsis AtMPKs and AtMKKs were used as queries to search for putative MPK and MKK proteins against the watermelon genome database at http:// www.icugi.org/. The obtained nucleotide and protein sequences were examined by domain analysis programs PFAM (http://pfam.sanger.ac.uk/) and SMART (http:// smart.emblheidelberg.de/) with the default cutoff parameters. The isoelectric points and molecular weights were predicted on the ExPASy Proteomics Server (http:// expasy.org/). Sequence alignment was carried out by the ClustalX program. Phylogenetic tree was constructed using the neighbor-joining method of the MEGA6 program with the $\mathrm{p}$-distance and complete deletion option parameters. The reliability of the obtained trees was tested using a bootstrapping method with 1000 replicates.

\section{Cloning of the CIMPK and CIMKK genes}

Total RNA was extracted by Trizol regent and treated with RNase-free DNase (TaKaRa, Dalian, China) according to the manufacturer's instructions. First-strand cDNA was synthesized using AMV reverse transcriptase (Takara, Dalian, China) with oligo $\mathrm{d}(\mathrm{T})$ primer according 
Table 2 Summary on the expression, protein-protein interaction, functions in disease resistance and putative Arabidopsis orthologs for the CIMPK and CIMKK genes

\begin{tabular}{|c|c|c|c|c|c|c|c|c|c|c|c|}
\hline \multirow[t]{3}{*}{ Genes } & \multicolumn{8}{|c|}{ Expression patterns } & \multirow{3}{*}{$\begin{array}{l}\text { Protein- } \\
\text { protein } \\
\text { interaction }^{c}\end{array}$} & \multirow{3}{*}{$\begin{array}{l}\text { Functions } \\
\text { in disease } \\
\text { resistance }^{d}\end{array}$} & \multirow{3}{*}{$\begin{array}{l}\text { Homolog in } \\
\text { Arabidopsis }\end{array}$} \\
\hline & \multicolumn{3}{|c|}{ Tissues $^{a}$} & \multicolumn{4}{|c|}{ Abiotic stress $^{b}$} & \multirow{2}{*}{$\frac{\text { Biotic stress }^{b}}{\text { Fon }}$} & & & \\
\hline & Root & Stem & Leaf & $\overline{\mathrm{Dr}}$ & $\mathrm{Sa}$ & $\mathrm{He}$ & $\overline{\mathrm{Co}}$ & & & & \\
\hline CIMPK1 & +++ & + & ++ & $\uparrow$ & $\uparrow$ & $\uparrow$ & $\downarrow$ & - & CIMKK2-2 & Increased & AtMPK1 \\
\hline CIMPK3 & + & +++ & + & $\uparrow$ & $\uparrow$ & $\uparrow$ & - & $\uparrow$ & Not studied & WT & AtMPK3 \\
\hline \multirow[t]{2}{*}{ CIMPK4-1 } & + & + & + & $\uparrow$ & $\uparrow$ & $\uparrow$ & $\downarrow$ & $\uparrow$ & CIMKK2-1 & WT & AtMPK4 \\
\hline & & & & & & & & & CIMKK6 & & \\
\hline \multirow[t]{2}{*}{ CIMPK4-2 } & + & + & + & $\uparrow$ & $\uparrow$ & $\uparrow$ & - & $\uparrow$ & CIMKK2-2 & Increased & AtMPK4 [52] \\
\hline & & & & & & & & & CIMKK6 & & \\
\hline CIMPK6 & + & + & + & $\uparrow$ & - & $\uparrow$ & $\uparrow$ & $\uparrow$ & CIMKK5 & Decreased & AtMPK6 $[55,56]$ \\
\hline \multirow[t]{2}{*}{ CIMPK7 } & +++ & + & + & $\uparrow$ & $\uparrow$ & $\uparrow$ & $\downarrow$ & $\uparrow$ & CIMKK5 & Increased & AtMPK7 $[50,58]$ \\
\hline & & & & & & & & & & HR-like cell death & \\
\hline CIMPK9-1 & +++ & + & +++ & $\uparrow$ & - & - & $\downarrow$ & $\uparrow$ & Not studied & Not studied & AtMPK9 \\
\hline CIMPK9-2 & + & + & ++ & $\uparrow$ & $\uparrow$ & - & $\downarrow$ & $\uparrow$ & - & Not studied & AtMPK9 \\
\hline CIMPK9-3 & + & + & ++ & - & - & $\uparrow$ & - & $\uparrow$ & Not studied & Not studied & AtMPK9 \\
\hline CIMPK9-4 & + & + & +++ & - & - & $\uparrow$ & $\downarrow$ & - & Not studied & Not studied & AtMPK9 \\
\hline \multirow[t]{2}{*}{ CIMPK13 } & ++ & ++ & + & $\uparrow$ & - & - & - & $\uparrow$ & CIMKK2-1 & WT & AtMPK13 \\
\hline & & & & & & & & & CIMKK6 & & \\
\hline CIMPK16 & + & + & +++ & $\uparrow$ & $\uparrow$ & - & - & $\uparrow$ & - & Not studied & AtMPK16 \\
\hline CIMPK19 & + & + & +++ & $\uparrow$ & $\uparrow$ & - & $\uparrow$ & $\uparrow$ & Not studied & WT & AtMPK19 \\
\hline CIMPK20-1 & + & +++ & +++ & $\uparrow$ & $\uparrow$ & $\uparrow$ & - & $\uparrow$ & Not studied & Not studied & AtMPK20 \\
\hline CIMPK20-2 & ++ & + & +++ & $\uparrow$ & - & $\uparrow$ & - & - & Not studied & Not studied & AtMPK20 \\
\hline \multirow[t]{3}{*}{ CIMKK2-1 } & +++ & ++ & + & $\uparrow$ & - & $\uparrow$ & - & $\uparrow$ & CIMPK4-1 & WT & AtMKK2 \\
\hline & & & & & & & & & CIMPK4-2 & & \\
\hline & & & & & & & & & CIMPK13 & & \\
\hline CIMKK2-2 & + & ++ & ++ & $\uparrow$ & $\uparrow$ & $\uparrow$ & - & $\uparrow$ & CIMPK1 & Decreased & AtMKK2 [45] \\
\hline CIMKK3 & + & ++ & + & $\uparrow$ & $\uparrow$ & $\uparrow$ & $\downarrow$ & $\uparrow$ & Not studied & Not studied & AtMKK3 \\
\hline \multirow[t]{2}{*}{ CIMKK5 } & + & +++ & + & $\uparrow$ & $\uparrow$ & $\uparrow$ & $\uparrow$ & $\uparrow$ & CIMPK6 & WT & AtMKK5 \\
\hline & & & & & & & & & CIMPK7 & & \\
\hline \multirow[t]{3}{*}{ CIMKK6 } & +++ & +++ & + & $\downarrow$ & - & - & $\uparrow$ & $\uparrow$ & CIMPK4-1 & WT & AtMKK6 \\
\hline & & & & & & & & & CIMPK4-2 & & \\
\hline & & & & & & & & & CIMPK13 & & \\
\hline CIMKK9 & ++ & ++ & ++ & - & - & - & - & $\uparrow$ & Not studied & Not studied & AtMKK9 \\
\hline
\end{tabular}

\footnotetext{
a represents the relative expression levels

${ }^{\mathrm{b}} \uparrow$ represents significant upregulation; $\downarrow$ indicates significant downregulation;-indicates no significant change. Dr, drought stress; Sa, salt stress; He, heat stress; Co, cold stress. Fon, Fusarium oxypsorum f. sp. niveum

'Putative interacting partners from yeast two hybrid assays are listed.-indicates no interacting partner was identified. Not studied, these CIMPKs or CIMKKs were not examined

${ }^{d}$ Possible functions of the selected CIMPKs and CIMKKs was examined using transient expression-based functional analysis in N. benthamiana. Increased, increased resistance against $B$. cinerea when transiently expressed in $N$. benthamiana; Decreased, decreased resistance against $B$. cinerea when transiently expressed in $N$. benthamiana. WT, wild-type phenotype
}

to the manufacturer's instructions. The obtained cDNAs were used for qRT-PCR and cloning. The coding sequences for $C l M P K s$ and $C l M K K s$ were amplified using gene-specific primers (Additional file 1: Table S1) designed based on the predicated cDNAs and cloned into pMD19-T vector via T/A cloning, yielding pMD19CIMPKs or pMD19-ClMKKs. After confirmation by sequencing, these pMD19-ClMPKs or pMD19-ClMKKs plasmids were used as templates to amplify the target genes for further experiments.

\section{Yeast two-hybrid assays}

Putative interactions between ClMPKs and ClMKKs were examined using the Matchmaker Gold Yeast Two- 
Hybrid System according to the manufacturer's instructions (Clontech, Mountain View, CA, USA). The coding sequences of ClMPKs and ClMKKs were amplified using gene-specific primers (Additional file 1: Table S1) from pMD19-ClMPKs or pMD19-ClMKKs and cloned into pGADT7 and pGBKT7 vectors. The resultant plasmids were transformed into yeast strains Y2HGold and confirmed by colony PCR. The transformed yeasts were cultivated on $\mathrm{SD} / \mathrm{Trp}^{-} \mathrm{His}^{-}$medium for 3 days at $30{ }^{\circ} \mathrm{C}$, followed by addition of X- $\alpha$-gal (5-Bromo-4chloro-3indolyl-a-D-galactopyranoside). Interactions between CIMPKs and ClMKKs were evaluated according to the growth situation of the transformed yeast cells on the $\mathrm{SD} / \mathrm{Trp}^{-} \mathrm{His}^{-}$medium and the production of blue pigments after the addition of X- $\alpha-G a l$. Co-transformation of pGBKT7-53 or pGBKT7-Lam and pGADT7-T were as positive and negative controls, respectively.

\section{Transient expression in $N$. benthamiana and disease assays}

The coding sequences of the selected ClMPKs and ClMKKs were amplified using gene-specific primers (Additional file 1: Table S1) from the corresponding pMD19-ClMPKs or pMD19-ClMKKs plasmids and cloned into pFGC-Egfp at different restriction enzyme sites, yielding pFGC-CIMPKs or pFGC-ClMKKs. The recombinant plasmids pFGC-ClMPKs or pFGC-ClMKKs and the empty vector pFGC-Egfp were introduced into Agrobacterium tumefaciens strain GV3101 by electroporation using GENE PULSER II Electroporation System (Bio-Rad Laboratories, Hercules, CA, USA). Agrobacteria carrying pFGC-ClMPKs, pFGC-ClMKKs or pFGCEgfp were grown in YEP medium $(50 \mu \mathrm{g} / \mathrm{ml}$ rifampicin, $50 \mu \mathrm{g} / \mathrm{ml}$ kanamycin and $25 \mu \mathrm{g} / \mathrm{ml}$ gentamicin) for $24 \mathrm{~h}$ with continuous shaking at $28{ }^{\circ} \mathrm{C}$, collected by centrifugation and resuspended in infiltration buffer $(10 \mathrm{mM}$ $\mathrm{MgCl}_{2}, 10 \mathrm{mM}$ MES, $200 \mu \mathrm{M}$ acetosyringone, pH5.7). For transient expression, agrobacteria carrying different constructs were infiltrated into leaves of 4-week-old $N$. benthamiana plants using $1 \mathrm{~mL}$ needleless syringes. Leaf samples were collected 2 days after agroinfiltration for analyzing the expression level of the target genes by qRT-PCR, level of protein accumulation by Western blot or for disease assays.

For disease assays, inoculation of $B$. cinerea was performed using spore suspension $\left(1 \times 10^{5}\right.$ spores $\left./ \mathrm{mL}\right)$ according to previously reported procedure [16]. Briefly, detached leaves were inoculated by dropping a $5 \mu \mathrm{L}$ of spore suspension and then kept in sealed trays at $22{ }^{\circ} \mathrm{C}$ to facilitate disease development. Disease progress was estimated by measuring the lesion sizes and fungal growth by qRT-PCR analyzing the transcript of $B$. cinerea ActinA gene as an indicative of fungal growth
$[16,61]$ using a pair of primers BcActin-F and BcActin$\mathrm{R}$ (Additional file 1: Table S1).

For Western blot analysis of the CIMPK7 protein, leaf discs were ground in $200 \mu \mathrm{L}$ lysis buffer (50 mM Tris$\mathrm{HCl}, \mathrm{pH} 7.4,150 \mathrm{mM} \mathrm{NaCl}, 1 \mathrm{mM}$ EDTA, $1 \mathrm{mM}$ DDT, $0.1 \%$ Triton $\mathrm{X}-100$, and $1 \times$ protease inhibitor cocktail, $1 \mathrm{mM}$ PMSF), followed by addition of $100 \mu \mathrm{L}$ loading buffer. After boiling for $5 \mathrm{~min}$, the samples were centrifuged at $10,000 \times g$ for $10 \mathrm{~min}$ at $4{ }^{\circ} \mathrm{C}$ and $20 \mu \mathrm{L}$ of the supernatant were separated on a $12 \%$ SDS-PAGE gel, followed by transferring onto PVDF membrane by semidry transfer. Detection of GFP was performed using a polyclonal rabbit anti-GFP antibody (1:5000 dilution; GenScript, Nanjing, China) and a Horseradish peroxidaseconjugated anti-rabbit antibody (1:10,000 dilution; GenScript, Nanjing, China) according to the manufacturer's instructions. Proteins in SDS-PAGE gel were detected by SuperSignal West Pico Chemiluminescent Substrate (Thermo Scientific, Rockford, IL, USA).

\section{Detection of $\mathrm{H}_{2} \mathrm{O}_{2}$ accumulation}

Detection of $\mathrm{H}_{2} \mathrm{O}_{2}$ was performed by DAB staining [62]. Leaf samples were collected from $N$. benthamiana plants at $48 \mathrm{~h}$ after infiltration for transient expression and dipped into DAB solution (1 mg/mL, pH3.8). After incubation for $8 \mathrm{~h}$ in dark at room temperature, the DABtreated leaves were transferred into acetic acid/glycerol/ ethanol (1:1:1, vol/vol/vol) and boiled for $5 \mathrm{~min}$, followed by several washes with the same solution. The DABstained leaves were photographed using a digital camera.

\section{qRT-PCR analysis of gene expression}

Total RNA was extracted by Trizol regent (TaKaRa, Dalian, China) according to the manufacturer's instructions. RNA was treated with RNase-free DNase and then reverse-transcribed into cDNA using the PrimeScript RT regent kit (TaKaRa, Dalian, China). The obtained cDNAs were used for gene expression analysis with real time quantitative PCR. Each qPCR reaction contained $12.5 \mu \mathrm{L}$ SYBR Premix Ex Taq (TaKaRa, Dalian, China), $0.1 \mu \mathrm{g}$ cDNA and 7.5 pmol of each gene-specific primer (Additional file 1: Table S1) in a final volume of $25 \mu \mathrm{L}$, and had three independent biological replicates. The qPCR was performed in a CFX96 real-time PCR detection system (Bio-Rad, Hercules, CA, USA). Relative gene expression level was calculated using $2^{-\triangle \Delta C T}$ method as described.

\section{Statistical analysis}

All experiments were repeated independently three times and data obtained from three independent experiments were subjected to statistical analysis according to the Student's $t$-test. The probability values of $p \leq 0.05$ 
were considered as significant difference between the treatments and corresponding controls.

\section{Availability of supporting data}

Sequence information on the watermelon and Arabidopsis MPKs and MKKs used in phylogenetic trees can be found in the LabArchives database under DOI of 10.6070/H4HQ3WXB (https://mynotebook.labarchives.com/share/Dayong\%2520Li/MjYuMHwxMDIwMDkvMj AvVHJlZU5vZGUvNzg5MzI4ODZ8NjYuMA==).

\section{Additional file}

Additional file 1: Table S1. Primers used in this study for different purposes. (DOC $136 \mathrm{~kb}$ )

\section{Abbreviations \\ ABA: Abscisic acid; B. cinerea: Botrytis cinerea; DAB: 3, 3-diaminobenzidine; dpi: Days after inoculation; Fon: Fusarium oxypsorum f. sp. niveum; HR: Hypersensitive response; MAPK: Mitogen-activated protein kinase: MKK: MAPK kinase: MEKK: MKK kinase: N. benthamiana: Nicotiana benthamiana; ORF: Open reading frame; qRT-PCR: Quantitative reverse transcription PCR.}

\section{Competing interests}

The authors declare that they have no competing interests.

\section{Authors' contributions}

Experiments were designed by FS, HZ, DL, QS. Experiments were performed by QS, YD, LH, SL, YH and HZ. FS, DL, HZ and QS drafted the manuscript and FS revised the manuscript. All authors read and approved the final manuscript.

\section{Acknowledgements}

The present study was financially supported by grants from the Fund for Modern Agro-industry Technology Research System (CARS-26-11) and the Priority Development Program of the Specialized Research Fund for the Doctoral Program of Higher Education (20130101130006)

Received: 20 April 2015 Accepted: 13 December 2015

Published online: 23 December 2015

\section{References}

1. Meng X, Zhang S. MAPK cascades in plant disease resistance signaling Annu Rev Phytopathol. 2013;51:245-66.

2. $\mathrm{Xu} \mathrm{J}$, Zhang S. Mitogen-activated protein kinase cascades in signaling plant growth and development. Trends Plant Sci. 2015;20(1):56-64.

3. Jonak C, Okresz L, Bogre L, Hirt H. Complexity, cross talk and integration of plant MAP kinase signalling. Curr Opin Plant Biol. 2002;5(5):415-24.

4. Suarez-Rodriguez MC, Petersen M, Mundy J. Mitogen-activated protein kinase signaling in plants. Annu Rev Plant Biol. 2010;61:621-49.

5. Colcombet J, Hirt H. Arabidopsis MAPKs: a complex signaling network involved in multiple biological processes. Biochem J. 2008;413(2):217-26.

6. Rasmussen MW, Roux M, Petersen M, Mundy J. MAP kinase cascades in Arabidopsis innate immunity. Front Plant Sci. 2012;3:169.

7. Pitzschke A, Schikora A, Hirt H. MAPK cascade signalling networks in plant defence. Curr Opin Plant Biol. 2009;12(4):421-6.

8. Ichimura K, Shinozaki K, Tena G, Sheen J, Henry Y, Zhang S. Mitogenactivated protein kinase cascades in plants: a new nomenclature. Trends Plant Sci. 2002;7(7):301-8.

9. Hamel LP, Nicole MC, Sritubtim S, Morency MJ, Ellis M, Ehlting J, et al. Ancient signals: comparative genomics of plant MAPK and MAPKK gene families. Trends Plant Sci. 2006:11(4):192-8.

10. Reyna NS, Yang Y. Molecular analysis of the rice MAP kinase gene family in relation to Magnaporthe grisea infection. Mol Plant Microbe Interact. 2006;19(5):530-40
11. Neupane A, Nepal MP, Piya S, Subramanian S, Rohila JS, Reese RN, et al. dentification, nomenclature, and evolutionary relationships of mitogenactivated protein kinase (MAPK) genes in soybean. Evol Bioinforma. 2013;9:363-86

12. Kong X, Pan J, Zhang D, Jiang S, Cai G, Wang L, et al. Identification of mitogenactivated protein kinase kinase gene family and MKK-MAPK interaction network in maize. Biochem Biophys Res Commun. 2013;441(4):964-9.

13. Liu Y, Zhang D, Li W, Li D. Genome-wide analysis of mitogen activated protein kinase gene family in maize. Plant Mol Biol Rep. 2013;31(6):1446-60.

14. Kong FL, Wang J, Cheng L, Liu S, Wu J, Peng Z, et al. Genome-wide analysis of the mitogen-activated protein kinase gene family in Solanum lycopersicum. Gene. 2012;499(1):108-20.

15. Wu J, Wang J, Pan C, Guan X, Wang Y, Liu S, et al. Genome-wide identification of MAPKK and MAPKKK gene families in tomato and transcriptional profiling analysis during development and stress response. PLoS One. 2014;9(7):e103032

16. Li X, Zhang Y, Huang L, Ouyang Z, Hong Y, Zhang H, et al. Tomato SIMKK2 and SIMKK4 contribute to disease resistance against Botrytis cinerea. BMC Plant Biol. 2014;14:166.

17. Liang W, Yang B, Yu BJ, Zhou Z, Li C, Jia M, et al. Identification and analysis of MKK and MPK gene families in canola (Brassica napus L.). BMC Genomics. 2013;14:392.

18. Asif MH, Lakhwani D, Pathak S, Bhambhani S, Bag SK, Trivedi PK. Genome-wide identification and expression analysis of the mitogen-activated protein kinase gene family from banana suggest involvement of specific members in different stages of fruit ripening. Funct Integr Genomics. 2014;14(1):161-75.

19. Zhang S, Xu R, Luo X, Jiang Z, Shu H. Genome-wide identification and expression analysis of MAPK and MAPKK gene family in Malus domestica. Gene. 2013;531(2):377-87.

20. Zhang X, Wang L, Xu X, Cai C, Guo W. Genome-wide identification of mitogen-activated protein kinase gene family in Gossypium raimondii and the function of their corresponding orthologs in tetraploid cultivated cotton. BMC Plant Biol. 2014;14(1):345

21. Wei C, Liu X, Long D, Guo Q, Fang Y, Bian C, et al. Molecular cloning and expression analysis of mulberry MAPK gene family. Plant Physiol Biochem. 2014;77(1):108-16.

22. Chen L, Hu W, Tan S, Wang M, Ma Z, Zhou S, et al. Genome-wide identification and analysis of MAPK and MAPKK gene families in Brachypodium distachyon. PLoS One. 2012;7:e46744.

23. Hyun TK, Kim JS, Kwon SY, Kim SH. Comparative genomic analysis of mitogen activated protein kinase gene family in grapevine. Genes Genomics. 2010;32(3):275-81.

24. Zhang X, Cheng T, Wang G, Yan Y, Xia Q. Cloning and evolutionary analysis of tobacco MAPK gene family. Mol Biol Rep. 2013:40(2):1407-15.

25. Steichen JM, Kuchinskas M, Keshwani M, Yang J, Adams JA, Taylor SS. Structural basis for the regulation of protein kinase $A$ by activation loop phosphorylation. J Biol Chem. 2012;287(18):14672-80.

26. Mohanta TK, Arora PK, Mohanta N, Parida P, Bae H. Identification of new members of the MAPK gene family in plants shows diverse conserved domains and novel activation loop variants. BMC Genomics. 2015;16:58.

27. Janitza P, Ullrich KK, Quint M. Toward a comprehensive phylogenetic reconstruction of the evolutionary history of mitogen-activated protein kinases in the plant kingdom. Front Plant Sci. 2012;3:271.

28. Guo S, Zhang J, Sun H, Salse J, Lucas WJ, Zhang H, et al. The draft genome of watermelon (Citrullus lanatus) and resequencing of 20 diverse accessions. Nat Genet. 2013;45(1):51-8.

29. Houb EB. The arms race is ancient history in Arabidopsis, the wildflower. Nat Rev Genetics. 2001:2(7):516-27.

30. Danquah A, de Zelicourt A, Colcombet J, Hirt H. The role of ABA and MAPK signaling pathways in plant abiotic stress responses. Biotechnol Adv. 2014; 32(1):40-52.

31. Moustafa K, AbuQamar S, Jarrar M, Al-Rajab AJ, Trémouillaux-Guiller J. MAPK cascades and major abiotic stresses. Plant Cell Rep. 2014;33(8):1217-25.

32. Liu Y. Roles of mitogen-activated protein kinase cascades in ABA signaling. Pant Cell Rep. 2012;31(1):1-12.

33. Mehrotra R, Bhalothia P, Bansal P, Basantani MK, Bharti V, Mehrotra S. Abscisic acid and abiotic stress tolerance - different tiers of regulation J Plant Physiol. 2014;171(7):486-96.

34. Martyn RD. Fusarium wilt of watermelon. In: Zitter TA, Hopkins DL, Thomas CE, editors. Compendium of cucurbit diseases. St. Paul: The American Phytopathology Society; 1996. p. 13-4. 
35. Michielse $C B$, Rep M. Pathogen profile update: Fusarium oxysporum. Mol Plant Pathol. 2009;10(3):311-24

36. Zhang $X$, Dai $Y$, Xiong $Y$, DeFraia $C$, Li J, Dong $X$, et al. Overexpression of Arabidopsis MAP kinase kinase 7 leads to activation of plant basal and systemic acquired resistance. Plant J. 2007;52(6):1066-79.

37. Fu ZQ, Dong X. Systemic acquired resistance: turning local infection into global defense. Annu Rev Plant Biol. 2013;64:839-63.

38. Zhang L, Li Y, Lu W, Meng F, Wu CA, Guo X. Cotton GhMKK5 affects disease resistance, induces HR-like cell death, and reduces the tolerance to salt and drought stress in transgenic Nicotiana benthamiana. J Exp Bot. 2012;63(10):3935-51.

39. Andreasson E, Ellis B. Convergence and specificity in the Arabidopsis MAPK nexus. Trends Plant Sci. 2010;15(2):106-13.

40. Tanoue T, Adachi M, Moriguchi T, Nishida E. A conserved docking motif in MAP kinases common to substrates, activators and regulators. Nat Cell Biol. 2000;2(2):110-6.

41. Asai T, Tena G, Plotnikova J, Willmann MR, Chiu WL, Gomez-Gomez L, et al. MAP kinase signalling cascade in Arabidopsis innate immunity. Nature. 2002;415(6875):977-83

42. Teige M, Scheikl E, Eulgem T, Dóczi R, Ichimura K, Shinozaki K, et al. The MKK2 pathway mediates cold and salt stress signaling in Arabidopsis. Mol Cell. 2004;15(1):141-52

43. Lee J, Huh K, Bhargava A, Ellis B. Comprehensive analysis of protein-protein interactions between Arabidopsis MAPKs and MAPK kinases helps define potential MAPK signalling modules. Plant Signal Behav. 2008;3(12):1037-41.

44. Popescu S, Popescu G, Bachan S, Zhang Z, Gerstein M, Snyder M, et al. MAPK target networks in Arabidopsis thaliana revealed using functional protein microarrays. Genes Dev. 2009;23(1):80-92.

45. Kong Q, Qu N, Gao M, Zhang Z, Ding X, Yang F, et al. The MEKK1-MKK1/ MKK2-MPK4 kinase cascade negatively regulates immunity mediated by a mitogen-activated protein kinase kinase kinase in Arabidopsis. Plant Cell. 2012:24(5):2225-36.

46. Takahashi Y, Soyano T, Kosetsu K, Sasabe M, Machida Y. HINKEL kinesin, ANP MAPKKKs and MKK6/ANQ MAPKK, which phosphorylates and activates MPK4 MAPK, constitute a pathway that is required for cytokinesis in Arabidopsis thaliana. Plant Cell Physiol. 2010;51(10):1766-76.

47. Kosetsu K, Matsunaga S, Nakagami H, Colcombet J, Sasabe M, Soyano T, et al. The MAP kinase MPK4 is required for cytokinesis in Arabidopsis thaliana. Plant Cell. 2010;22(11):3778-90.

48. Zeng $\mathrm{Q}$, Chen JG, Ellis BE. AtMPK4 is required for male-specific meiotic cytokinesis in Arabidopsis. Plant J. 2011;67(5):895-906.

49. Wankhede DP, Misra M, Singh P, Sinha AK. Rice mitogen activated protein kinase kinase and mitogen activated protein kinase interaction network revealed by in-silico docking and yeast two-hybrid approaches. PLoS One. 2013;8(5):e65011.

50. Dóczi R, Brader G, Pettkó-Szandtner A, Rajh I, Djamei A, Pitzschke A, et al. The Arabidopsis mitogen-activated protein kinase kinase MKK3 is upstream of group C mitogen-activated protein kinases and participates in pathogen signaling. Plant Cell. 2007;19(10):3266-79.

51. Shi J, An HL, Zhang L, Gao Z, Guo XQ. GhMPK7, a novel multiple stressresponsive cotton group C MAPK gene, has a role in broad spectrum disease resistance and plant development. Plant Mol Biol. 2010;74(1-2):1-17.

52. Brodersen P, Petersen M, Biørn-Nielsen H, Zhu S, Newman MA, Shokat KM et al. Arabidopsis MAP kinase 4 regulates salicylic acid- and jasmonic acid/ ethylenedependent responses via EDS1 and PAD4. Plant J. 2006:47(4):532-46.

53. Virk N, Liu B, Zhang H, Li X, Zhang Y, Li D, et al. Tomato SIMPK4 is required for resistance against Botrytis cinerea and tolerance to drought stress. Acta Physiol Plant. 2013;35(4):1211-21.

54. Wang Z, Mao H, Dong C, Ji R, Cai L, Fu H, et al. Overexpression of Brassica napus MPK4 enhances resistance to Sclerotinia sclerotiorum in oilseed rape. Mol Plant Microbe Interact. 2009;22(3):235-44.

55. Ren D, Liu Y, Yang KY, Han L, Mao G, Glazebrook J, et al. A fungal-responsive MAPK cascade regulates phytoalexin biosynthesis in Arabidopsis. Proc Natl Acad Sci U S A. 2008:105(14):5638-43.

56. Galletti R, Ferrari S, De Lorenzo G. Arabidopsis MPK3 and MPK6 play different roles in basal and oligogalacturonide- or flagellin-induced resistance against Botrytis cinerea. Plant Physiol. 2011;157(2):804-14.

57. Liu Y, Ren D, Pike S, Pallardy S, Gassmann W, Zhang S. Chloroplast-generated reactive oxygen species are involved in hypersensitive response-like cell death mediated by a mitogen-activated protein kinase cascade. Plant J. 2007;51(6): 941-54.
58. Ren D, Yang H, Zhang S. Cell death mediated by mitogen-activated protein kinase pathway is associated with the generation of hydrogen peroxide in Arabidopsis. J Biol Chem. 2002;277(1):559-65.

59. Ren D, Yang K-Y, Li G, Liu Y, Zhang S. Activation of Ntf4, a tobacco MAPK, during plant defense response and its involvement in hypersensitive response-like cell death. Plant Physiol. 2006;141(4):1482-93.

60. Zhou XG, Everts KL. Quantification of root and stem colonization of watermelon by Fusarium oxysporum f. sp. niveum and its use in evaluating resistance. Phytopathology. 2004;94(8):832-41.

61. Benito EP, Ten Have A, van't Klooster JW, Van Kan JAL. Fungal and plant gene expression during synchronized infection of tomato leaves by Botrytis cinerea. Eur J Plant Pathol. 1998;104(2):207-20.

62. Thordal-Christensen $H$, Zhang ZG, Wei YD, Collinge DB. Subcellular localization of $\mathrm{H}_{2} \mathrm{O}_{2}$ in plants. $\mathrm{H}_{2} \mathrm{O}_{2}$ accumulation in papillae and hypersensitive response during the barley-powdery mildew interaction. Plant J. 1997;11(6):1187-94.

\section{Submit your next manuscript to BioMed Central and we will help you at every step:}

- We accept pre-submission inquiries

- Our selector tool helps you to find the most relevant journal

- We provide round the clock customer support

- Convenient online submission

- Thorough peer review

- Inclusion in PubMed and all major indexing services

- Maximum visibility for your research

Submit your manuscript at www.biomedcentral.com/submit
Biomed Central 